\title{
Cauchy-Kowalevski extensions and monogenic plane waves using spherical monogenics
}

\author{
N. De Schepper ${ }^{a}{ }^{*}$ F. Sommen ${ }^{\mathrm{a}}$ \\ ${ }^{a}$ Clifford Research Group, Ghent University, Department of Mathematical \\ Analysis, Faculty of Engineering, Galglaan 2, B-9000 Gent, Belgium
}

\begin{abstract}
Clifford analysis may be regarded as a direct and elegant generalization to higher dimensions of the theory of holomorphic functions in the complex plane, centred around the notion of monogenic function, i.e. a null solution of the Dirac operator. This paper deals with axial and biaxial monogenic functions containing spherical monogenics. They are constructed by means of two fundamental methods of Clifford analysis, namely the Cauchy-Kowalevski extension and monogenic plane waves.
\end{abstract}

Key words: Clifford analysis, CK-extension, monogenic plane waves, axial monogenic function, biaxial monogenic function, Cauchy-Riemann equation 1991 MSC: 30G35, 33C05, 33C15, 33C45

\footnotetext{
* Corresponding author. Telephone: +32 (0)9 26449 21. Fax: +32 (0)9 2644987. Email addresses: nds@cage.ugent.be (N. De Schepper), fs@cage.ugent.be ( F. Sommen).
} 


\section{Introduction}

Clifford analysis (see a.o. [1,3]) is a theory that offers a natural generalization of complex analysis to higher dimensions. To $\mathbb{R}^{m}$, the Euclidean space in $m$ dimensions, we first associate the Clifford algebra $\mathcal{C} l_{0, m}$, generated by the canonical basis $e_{i}, i=1, \ldots, m$. These generators satisfy the multiplication rules $e_{i} e_{j}+e_{j} e_{i}=-2 \delta_{i j}$.

The Clifford algebra $\mathcal{C} l_{0, m}$ can be decomposed as $\mathcal{C} l_{0, m}=\oplus_{k=0}^{m} \mathcal{C} l_{0, m}^{k}$ with $\mathcal{C} l_{0, m}^{k}$ the space of $k$-vectors defined by $\mathcal{C} l_{0, m}^{k}=\operatorname{span}\left\{e_{i_{1} \ldots i_{k}}=e_{i_{1}} \ldots e_{i_{k}}, \quad i_{1}<\ldots<i_{k}\right\}$. More precisely, we have that the space of 1 -vectors is given by $\mathcal{C} l_{0, m}^{1}=\operatorname{span}\left\{e_{i}, i=1, \ldots, m\right\}$ and it is obvious that this space is isomorphic with $\mathbb{R}^{m}$. The space of so-called bivectors is given explicitly by $\mathcal{C} l_{0, m}^{2}=\operatorname{span}\left\{e_{i j}=e_{i} e_{j}, i<j\right\}$. Moreover, the even subalgebra $\mathcal{C} l_{0, m}^{+}$of $\mathcal{C} l_{0, m}$ is defined by $\mathcal{C} l_{0, m}^{+}=\sum_{k \text { even }} \oplus \mathcal{C} l_{0, m}^{k}$. Similarly, $\mathcal{C} l_{0, m}^{-}=\sum_{k \text { odd }} \oplus \mathcal{C} l_{0, m}^{k}$ is the so-called odd subalgebra.

We identify the point $\left(x_{1}, \ldots, x_{m}\right)$ in $\mathbb{R}^{m}$ with the vector variable $\underline{x}$ given by $\underline{x}=\sum_{j=1}^{m} x_{j} e_{j}$.

The Clifford product of two vectors splits into a scalar part and a bivector part:

$$
\underline{x} \underline{y}=\underline{x} \bullet \underline{y}+\underline{x} \wedge \underline{y}
$$

with

$$
\begin{aligned}
& \underline{x} \bullet \underline{y}=-\langle\underline{x}, \underline{y}\rangle=-\sum_{j=1}^{m} x_{j} y_{j}=\frac{1}{2}(\underline{x} \underline{y}+\underline{y} \underline{x}) \\
& \underline{x} \wedge \underline{y}=\sum_{j<k} e_{j} e_{k}\left(x_{j} y_{k}-x_{k} y_{j}\right)=\frac{1}{2}(\underline{x} \underline{y}-\underline{y} \underline{x}) .
\end{aligned}
$$

It is interesting to note that the square of a vector variable $\underline{x}$ is scalar-valued and equals the norm squared up to a minus sign: $\underline{x}^{2}=-\langle\underline{x}, \underline{x}\rangle=-|\underline{x}|^{2}$.

In a similar way we introduce a first order vector differential operator by $\partial_{\underline{x}}=\sum_{j=1}^{m} \partial_{x_{j}} e_{j}$. This operator is the so-called Dirac operator. Its square equals, up to a minus sign, the Laplace operator in $\mathbb{R}^{m}: \partial_{\underline{x}}^{2}=-\Delta_{m}$. In the sequel, we will also consider the so-called Cauchy-Riemann operator $\partial_{x_{0}}+\partial_{\underline{x}}$ for which $\left(\partial_{x_{0}}+\partial_{\underline{x}}\right)\left(\partial_{x_{0}}-\partial_{\underline{x}}\right)=\Delta_{m+1}$. A function 
$f\left(x_{1}, \ldots, x_{m}\right)$, respectively $f\left(x_{0}, x_{1}, \ldots, x_{m}\right)$, defined and continuously differentiable in an open domain of $\mathbb{R}^{m}$, respectively $\mathbb{R}^{m+1}$, is called monogenic in that region if $\partial_{\underline{x}} f=0$, respectively $\left(\partial_{x_{0}}+\partial_{\underline{x}}\right) f=0$. They generally take values in the full Clifford algebra $\mathcal{C} l_{0, m}$. The spinor spaces may be interpreted as minimal left ideals of $\mathcal{C} l_{0, m}$ of the form $S=\mathcal{C} l_{0, m} I$, $I$ being the primitive idempotent. Hence solutions of the spinor Dirac operator are in fact monogenic functions of the form $f I$. In this paper we only consider monogenic functions with values in the full multivector space $\mathcal{C} l_{0, m}$.

A monogenic homogeneous polynomial $P_{k}$ of degree $k(k \geq 0)$ in $\mathbb{R}^{m}$ is called a solid inner spherical monogenic of degree $k$. The set of all solid inner spherical monogenics of degree $k$ will be denoted by $\mathcal{M}_{k}$.

A monogenic function is called an axial monogenic of degree $k$ if it is of the form

$$
f\left(x_{0}, \underline{x}\right)=\left(A\left(x_{0},|\underline{x}|\right)+\frac{\underline{x}}{|\underline{x}|} B\left(x_{0},|\underline{x}|\right)\right) P_{k}(\underline{x}),
$$

where $A, B$ are scalar functions and $P_{k}$ is a solid inner spherical monogenic of degree $k$. The monogenicity condition $\left(\partial_{x_{0}}+\partial_{\underline{x}}\right) f=0$ then leads to the Vekua-type system for $A$ and $B$ :

$$
\partial_{x_{0}} A-\partial_{r} B=\frac{2 k+m-1}{r} B \quad, \quad \partial_{x_{0}} B+\partial_{r} A=0 \quad, \quad r=|\underline{x}|,
$$

the solution of which leads to numerous special monogenic functions (see [15]).

In general every monogenic function $f\left(x_{0}, \underline{x}\right)$ is determined by its restriction $f(0, \underline{x})$ to the hyperplane $x_{0}=0$ and conversely any given real analytic function $f(\underline{x})$ has a unique monogenic extension $f\left(x_{0}, \underline{x}\right)$ called Cauchy-Kowalevski extension (abbreviated CK-extension) (see [14]). Axial monogenics of degree $k$ are also determined by their restriction $\lim _{|\underline{x}| \rightarrow 0} \frac{1}{|\underline{x}|^{k}} f\left(x_{0}, \underline{x}\right)$, which turns out to be $A\left(x_{0}, 0\right) P_{k}(\underline{\omega})$ and consequently, starting from a given function $A\left(x_{0}\right)$ of one variable, one may establish its generalized axial CK-extension $\left(A\left(x_{0},|\underline{x}|\right)+\frac{\underline{x}}{|\underline{x}|} B\left(x_{0},|\underline{x}|\right)\right) P_{k}(\underline{x})$. This generalizes the standard CK-extension that is used in our former paper [4]. If one composes this operation with the restriction to $x_{0}=0$ we 
arrive at a correspondence

$$
A\left(x_{0}\right) \longrightarrow\left(A(0,|\underline{x}|)+\frac{\underline{x}}{|\underline{x}|} B(0,|\underline{x}|)\right) P_{k}(\underline{x})
$$

transforming one dimensional functions into higher dimensional ones. In Section 2 we study this generalized axial CK-extension and the resulting correspondence in detail for some cases, namely $A\left(x_{0}\right)=e^{x_{0}}, A\left(x_{0}\right)=e^{-x_{0}^{2} / 2}$ and $A\left(x_{0}\right)=\left(1-x_{0}^{2}\right)^{\alpha}, \alpha \in \mathbb{R}$.

In Section 3 we generalize our result to the case of biaxial monogenic functions. These functions originate from the more general splitting $\mathbb{R}^{m}=\mathbb{R}^{p} \oplus \mathbb{R}^{q}$ giving rise to two vector variables $\underline{x}=\sum_{j=1}^{p} x_{j} e_{j}, \underline{y}=\sum_{j=1}^{q} y_{j} e_{p+j}$ that anti-commute, and the corresponding Dirac operators $\partial_{\underline{x}}=\sum_{j=1}^{p} \partial_{x_{j}} e_{j}, \partial_{\underline{y}}=\sum_{j=1}^{q} \partial_{y_{j}} e_{p+j}$. The over-all Dirac operator is $\partial_{\underline{x}}+\partial_{\underline{y}}$ and biaxial monogenics are defined as solutions of $\left(\partial_{\underline{x}}+\partial_{\underline{y}}\right) f(\underline{x}, \underline{y})=0$ of the special form $\left(\underline{x}=r \underline{\omega}, \underline{y}=\rho \underline{\xi}, \underline{\omega} \in S^{p-1}, \underline{\xi} \in S^{q-1}\right)$

$$
(A(r, \rho)+\underline{\omega} B(r, \rho)+\underline{\xi} C(r, \rho)+\underline{\omega} \underline{\xi} D(r, \rho)) P_{k, \ell}(\underline{\omega}, \underline{\xi})
$$

whereby $A, B, C, D$ are scalar functions and $P_{k, \ell}$ is a solid inner spherical monogenic of degree $k, \ell$ in the variables $\underline{x}$ and $\underline{y}$, i.e. $P_{k, \ell}(\lambda \underline{x}, \mu \underline{y})=\lambda^{k} \mu^{\ell} P_{k, \ell}(\underline{x}, \underline{y})$ and $\partial_{\underline{x}}\left[P_{k, \ell}(\underline{x}, \underline{y})\right]=$ $\partial_{\underline{y}}\left[P_{k, \ell}(\underline{x}, \underline{y})\right]=0$. An example of such a solid inner spherical monogenic of degree $k, \ell$ is $P_{k, \ell}(\underline{x}, \underline{y})=P_{k}(\underline{x}) P_{\ell}(\underline{y})$ with $P_{k}$, respectively $P_{\ell}$, a solid inner spherical monogenic of degree $k$, respectively $\ell$, in $\underline{x}$, respectively $\underline{y}$. Moreover it may be shown that (see [3]) the monogenicity condition leads to Vekua-type systems for the pairs $(A, D)$ and $(B, C)$ :

$$
\left(\partial_{r}-\frac{k}{r}\right) A+\left(\partial_{\rho}+\frac{\ell+q-1}{\rho}\right) D=0, \quad\left(\partial_{r}+\frac{k+p-1}{r}\right) D-\left(\partial_{\rho}-\frac{\ell}{\rho}\right) A=0
$$

respectively

$$
\left(\partial_{r}+\frac{k+p-1}{r}\right) B+\left(\partial_{\rho}+\frac{\ell+q-1}{\rho}\right) C=0, \quad\left(\partial_{r}-\frac{k}{r}\right) C-\left(\partial_{\rho}-\frac{\ell}{\rho}\right) B=0 .
$$

Similar to axial monogenics, biaxial monogenics are determined by their restriction to the first axis $\mathbb{R}^{p}$ given by $\underline{y}=\underline{0}$ and the second axis $\mathbb{R}^{q}$ given by $\underline{x}=\underline{0}$. This leads to a 
correspondence between functions on $\mathbb{R}^{p}$ and functions on $\mathbb{R}^{q}$ that will be investigated for a number of examples.

In the fourth section we will produce special axial and biaxial monogenics as linear superpositions of plane wave type monogenic functions.

In the axial case we start from monogenic plane waves of the simple form $h\left(x_{0}+i\langle\underline{x}, \underline{t}\rangle\right)(1-$ $i \underline{t})$, whereby $\underline{t} \in S^{m-1}$ is a variable unit vector and $h$ is a classical holomorphic function of the complex variable $x_{0}+i\langle\underline{x}, \underline{t}\rangle$. We then consider the integral

$$
\int_{S^{m-1}} h\left(x_{0}+i\langle\underline{x}, \underline{t}\rangle\right)(1-i \underline{t}) P_{k}(\underline{t}) d \underline{t}
$$

$P_{k}$ being a fixed inner spherical monogenic of degree $k$, which may be evaluated using Funk-Hecke's theorem and gives, up to a constant, rise to the generalized axial CKextension of $h^{(k)}\left(x_{0}\right)$ (see Theorem 4.2). Note that in case of the function $h\left(x_{0}\right)=e^{x_{0}}$ we can link this approach with the generalized axial CK-extension technique of Section 2, since then $h^{(k)}\left(x_{0}\right)=h\left(x_{0}\right)$ (see subsection 4.2).

In the biaxial case we start from plane wave functions $F(\underline{x}, \underline{y} ; \underline{t}, \underline{s})$ of either form $h(i\langle\underline{x}, \underline{t}\rangle-$ $\langle\underline{y}, \underline{s}\rangle)(\underline{t}+i \underline{s})$ or $h(i\langle\underline{x}, \underline{t}\rangle-\langle\underline{y}, \underline{s}\rangle)(1+i \underline{t s})$ with $\underline{t} \in S^{p-1}, \underline{s} \in S^{q-1}$ variable orthogonal unit vectors. After integration over the bi-sphere $(\underline{t}, \underline{s}) \in S^{p-1} \times S^{q-1}$ of $F(\underline{x}, \underline{y} ; \underline{t}, \underline{s}) P_{k, \ell}(\underline{t}, \underline{s})$ with $P_{k, \ell}$ an even Clifford algebra-valued inner spherical monogenic of degree $k, \ell$ in the variable $\underline{x}$ and $\underline{y}$, we obtain biaxial monogenics of odd and of even type respectively. In subsection 4.3 we work out these integrals explicitly for the exponential function $\exp (i\langle\underline{x}, \underline{t}\rangle-\langle\underline{y}, \underline{s}\rangle)$, giving rise to integral formulas for the Clifford-Bessel function of biaxial type. The methods used in this section are substantially more complicated than those in [4], due to the use of Funk-Hecke's formula in its most general form.

It is clear that this paper deals with simple, though fundamental, examples of special monogenic functions and that many more special functions may be computed using the described methods. 
For an introduction to Clifford analysis we refer to $[1,3,5,6]$. Other papers dealing with axial and biaxial monogenics are $[2,8,9,11-13]$.

This paper generalizes the results obtained in [4]. For the sake of readability, we have repeated some formulae and calculations, which is then explicitly mentioned.

\section{Generalized Cauchy-Kowalevski extensions: the axial framework}

\subsection{General problem}

We start with explaining the idea of generalized axial CK-extensions.

Let us consider an $S O(m)$-invariant domain $\widetilde{\Omega} \subset \mathbb{R}^{m+1}$ and let us assume that the intersections of $\widetilde{\Omega}$ with all subspaces parallel to $\mathbb{R}^{m}$ are convex. Putting $\Omega=\widetilde{\Omega} \cap \mathbb{R}$, we have the following result (see [3]).

Theorem 2.1 Let $P_{k}(\underline{x}) \in \mathcal{M}_{k}$ be fixed and let $W_{0}\left(x_{0}\right)$ be an analytic function in $\Omega$. Then there exists a unique sequence $\left(W_{s}\left(x_{0}\right)\right)_{s>0}$ of analytic functions such that the series

$$
f\left(x_{0}, \underline{x}\right)=\sum_{s=0}^{\infty} \underline{x}^{s} W_{s}\left(x_{0}\right) P_{k}(\underline{x})
$$

is convergent in a neighbourhood $U \subset \mathbb{R}^{m+1}$ of the domain $\Omega$ and its sum $f$ is monogenic in $U$. The function $W_{0}\left(x_{0}\right)$ is determined by the relation

$$
P_{k}(\underline{\omega}) W_{0}\left(x_{0}\right)=\lim _{|\underline{x}| \rightarrow 0} \frac{1}{|\underline{x}|^{k}} f\left(x_{0}, \underline{x}\right), \quad \underline{\omega}=\frac{\underline{x}}{|\underline{x}|} \in S^{m-1} .
$$

The series $f\left(x_{0}, \underline{x}\right)$ is the generalized axial CK-extension of the function $W_{0}\left(x_{0}\right)$.

In this section we consider series of the form

$$
f\left(x_{0}, \underline{x}\right)=\sum_{s=0}^{\infty} c_{s, k} \underline{x}^{s} F_{s, k}\left(x_{0}\right) W\left(x_{0}\right) P_{k}(\underline{x})
$$


with $F_{0, k}\left(x_{0}\right)=1$, the coefficients $c_{s, k}$ satisfying

$$
\partial_{\underline{x}}\left[c_{s, k} \underline{x}^{s} P_{k}(\underline{x})\right]=c_{s-1, k} \underline{x}^{s-1} P_{k}(\underline{x}), \quad c_{0, k}=1
$$

and $\left(\partial_{x_{0}}+\partial_{\underline{x}}\right)\left[f\left(x_{0}, \underline{x}\right)\right]=0$. In other words, $f$ is monogenic in the whole of $\mathbb{R}^{m+1}$ and

$$
\lim _{|\underline{x}| \rightarrow 0} \frac{1}{|\underline{x}|^{k}} f\left(x_{0}, \underline{x}\right)=P_{k}(\underline{\omega}) W\left(x_{0}\right), \quad \underline{\omega}=\frac{\underline{x}}{|\underline{x}|} \in S^{m-1},
$$

i.e. $f\left(x_{0}, \underline{x}\right)$ is the generalized axial CK-extension of $W\left(x_{0}\right)$. The aim is to determine

$$
f(0, \underline{x})=\left(\sum_{s=0}^{\infty} c_{s, k} \underline{x}^{s} F_{s, k}(0) W(0)\right) P_{k}(\underline{x}) .
$$

By means of induction and using the fundamental formula

$$
\partial_{\underline{x}}\left[\underline{x}^{s} P_{k}(\underline{x})\right]= \begin{cases}-s \underline{x}^{s-1} P_{k}(\underline{x}) & \text { for } s \text { even } \\ -(s-1+m+2 k) \underline{x}^{s-1} P_{k}(\underline{x}) & \text { for } s \text { odd }\end{cases}
$$

one can prove that the coefficients $c_{s, k}$ satisfying (1), take the form

$$
c_{2 \ell, k}=\frac{\Gamma\left(\frac{m}{2}+k\right)}{\Gamma\left(\frac{m}{2}+k+\ell\right) 2^{2 \ell} \ell !} \quad \text { and } \quad c_{2 \ell+1, k}=-\frac{\Gamma\left(\frac{m}{2}+k\right)}{\Gamma\left(\frac{m}{2}+k+\ell+1\right) 2^{2 \ell+1} \ell !} .
$$

\subsection{Examples}

\subsection{1 $W\left(x_{0}\right)=e^{x_{0}}$}

This example was already considered in [15]. Putting

$$
f\left(x_{0}, \underline{x}\right)=\sum_{s=0}^{\infty} c_{s, k} \underline{x}^{s} B_{s, k}\left(x_{0}\right) e^{x_{0}} P_{k}(\underline{x})
$$

with $B_{0, k}\left(x_{0}\right)=1$, the coefficients $c_{s, k}$ satisfying (1) and $f$ being monogenic in $\mathbb{R}^{m+1}$, the aim is to determine $f(0, \underline{x})$. 
The first step is to obtain $B_{s, k}\left(x_{0}\right)$. Expressing the monogenicity of $f$, we find consecutively

$$
\begin{aligned}
& \left(\partial_{x_{0}}+\partial_{\underline{x}}\right)\left[\sum_{s=0}^{\infty} c_{s, k} \underline{x}^{s} B_{s, k}\left(x_{0}\right) e^{x_{0}} P_{k}(\underline{x})\right]=0 \\
& \Longleftrightarrow \sum_{s=0}^{\infty} B_{s, k}^{\prime}\left(x_{0}\right) e^{x_{0}} c_{s, k} \underline{x}^{s} P_{k}(\underline{x})+\sum_{s=0}^{\infty} B_{s, k}\left(x_{0}\right) e^{x_{0}} c_{s, k} \underline{x}^{s} P_{k}(\underline{x}) \\
& +\sum_{s=1}^{\infty} B_{s, k}\left(x_{0}\right) e^{x_{0}} c_{s-1, k} \underline{x}^{s-1} P_{k}(\underline{x})=0 \\
& \Longleftrightarrow \sum_{s=0}^{\infty} B_{s, k}^{\prime}\left(x_{0}\right) e^{x_{0}} c_{s, k} \underline{x}^{s} P_{k}(\underline{x})+\sum_{s=0}^{\infty} B_{s, k}\left(x_{0}\right) e^{x_{0}} c_{s, k} \underline{x}^{s} P_{k}(\underline{x}) \\
& +\sum_{s=0}^{\infty} B_{s+1, k}\left(x_{0}\right) e^{x_{0}} c_{s, k} \underline{x}^{s} P_{k}(\underline{x})=0
\end{aligned}
$$

where in the last series the substitution $s^{\prime}=s-1$ was executed. The functions $B_{s, k}\left(x_{0}\right)$ must hence satisfy the recurrence relation

$$
B_{s+1, k}\left(x_{0}\right)=-B_{s, k}\left(x_{0}\right)-B_{s, k}^{\prime}\left(x_{0}\right) \quad, \quad B_{0, k}\left(x_{0}\right)=1
$$

from which it follows that $B_{2 \ell, k}\left(x_{0}\right)=1$ and $B_{2 \ell+1, k}\left(x_{0}\right)=-1$.

Next, using spherical co-ordinates $\underline{x}=|\underline{x}| \underline{\omega}, \underline{\omega} \in S^{m-1}$, we obtain

$$
\begin{aligned}
f(0, \underline{x})=\sum_{s=0}^{\infty} c_{s, k} \underline{x}^{s} B_{s, k}(0) P_{k}(\underline{x}) & =\sum_{\ell=0}^{\infty} c_{2 \ell, k} \underline{x}^{2 \ell} P_{k}(\underline{x})-\sum_{\ell=0}^{\infty} c_{2 \ell+1, k} \underline{x}^{2 \ell+1} P_{k}(\underline{x}) \\
& =\Gamma\left(\frac{m}{2}+k\right)\left(\sum_{\ell=0}^{\infty} \frac{(-1)^{\ell}|\underline{x}|^{2 \ell}}{\Gamma\left(\frac{m}{2}+k+\ell\right) 2^{2 \ell} \ell !}\right) P_{k}(\underline{x}) \\
& +\Gamma\left(\frac{m}{2}+k\right) \frac{1}{2}\left(\sum_{\ell=0}^{\infty} \frac{(-1)^{\ell}|\underline{x}|^{2 \ell}}{\Gamma\left(\frac{m}{2}+k+\ell+1\right) 2^{2 \ell} \ell !}\right) \underline{x} P_{k}(\underline{x}) .
\end{aligned}
$$

In view of

$$
2^{\nu} \widetilde{J}_{\nu}(\rho)=\sum_{\ell=0}^{\infty} \frac{(-1)^{\ell} \rho^{2 \ell}}{\ell ! \Gamma(\nu+\ell+1) 2^{2 \ell}}
$$

with $\widetilde{J}_{\nu}(\rho)=\rho^{-\nu} J_{\nu}(\rho), J_{\nu}$ being the Bessel function of the first kind, we finally arrive at

$$
f(0, \underline{x})=\Gamma\left(\frac{m}{2}+k\right) 2^{m / 2+k-1}\left(\widetilde{J}_{m / 2+k-1}(|\underline{x}|)+\widetilde{J}_{m / 2+k}(|\underline{x}|) \underline{x}\right) P_{k}(\underline{x}) .
$$

Remark 2.1 Note that the full generalized axial CK-extension of $e^{x_{0}}$ is given by:

$$
f\left(x_{0}, \underline{x}\right)=\Gamma\left(\frac{m}{2}+k\right) 2^{m / 2+k-1}\left(\widetilde{J}_{m / 2+k-1}(|\underline{x}|)+\widetilde{J}_{m / 2+k}(|\underline{x}|) \underline{x}\right) e^{x_{0}} P_{k}(\underline{x}) .
$$




\subsection{2 $W\left(x_{0}\right)=e^{-x_{0}^{2} / 2}$}

We now consider the series

$$
f\left(x_{0}, \underline{x}\right)=\sum_{s=0}^{\infty} c_{s, k} \underline{x}^{s} H_{s, k}\left(x_{0}\right) e^{-x_{0}^{2} / 2} P_{k}(\underline{x})
$$

with $H_{0, k}\left(x_{0}\right)=1, f$ being monogenic in $\mathbb{R}^{m+1}$ and the coefficients $c_{s, k}$ given by (3). We again want to obtain a closed form for $f(0, \underline{x})$.

In a similar way as for the first example, the monogenicity of $f$ yields:

$$
H_{s+1, k}\left(x_{0}\right)=x_{0} H_{s, k}\left(x_{0}\right)-H_{s, k}^{\prime}\left(x_{0}\right) \quad, \quad H_{0, k}\left(x_{0}\right)=1 .
$$

From the above recurrence relation it follows that $H_{s, k}\left(x_{0}\right)$ are the classical Hermite polynomials on the real line associated with the weight function $e^{-x_{0}^{2} / 2}$, which are defined by the Rodrigues formula

$$
H_{s, k}\left(x_{0}\right)=e^{x_{0}^{2} / 2}\left(-\frac{d}{d x_{0}}\right)^{s}\left[e^{-x_{0}^{2} / 2}\right]
$$

These polynomials satisfy (see e.g. [10], p. 250):

$$
H_{2 \ell, k}(0)=\frac{(-1)^{\ell}(2 \ell) !}{\ell ! 2^{\ell}} \quad \text { and } \quad H_{2 \ell+1, k}(0)=0
$$

from which we obtain

$$
f(0, \underline{x})=\sum_{\ell=0}^{\infty} c_{2 \ell, k} \underline{x}^{2 \ell} H_{2 \ell, k}(0) P_{k}(\underline{x})=\sum_{\ell=0}^{\infty} \frac{\Gamma\left(\frac{m}{2}+k\right)}{\Gamma\left(\frac{m}{2}+k+\ell\right)} \frac{(2 \ell) ! 2^{-2 \ell}}{(\ell !)^{2}}\left(\frac{|\underline{x}|^{2}}{2}\right)^{\ell} P_{k}(\underline{x}) .
$$

Taking into account (see e.g. [10], p. 3)

$$
\frac{\Gamma\left(\ell+\frac{1}{2}\right)}{\Gamma\left(\frac{1}{2}\right)}=2^{-2 \ell} \frac{(2 \ell) !}{\ell !}
$$

we finally arrive at

$$
f(0, \underline{x})=\frac{\Gamma\left(\frac{m}{2}+k\right)}{\Gamma\left(\frac{1}{2}\right)} \sum_{\ell=0}^{\infty} \frac{\Gamma\left(\ell+\frac{1}{2}\right)}{\Gamma\left(\ell+\frac{m}{2}+k\right)} \frac{1}{\ell !}\left(\frac{|\underline{x}|^{2}}{2}\right)^{\ell} P_{k}(\underline{x})={ }_{1} F_{1}\left(\frac{1}{2} ; \frac{m}{2}+k ; \frac{|\underline{x}|^{2}}{2}\right) P_{k}(\underline{x})
$$


with

$$
{ }_{1} F_{1}(a ; c ; z)=\frac{\Gamma(c)}{\Gamma(a)} \sum_{\ell=0}^{\infty} \frac{\Gamma(a+\ell)}{\Gamma(c+\ell)} \frac{z^{\ell}}{\ell !}
$$

Kummer's function.

\subsection{3 $W\left(x_{0}\right)=\left(1-x_{0}^{2}\right)^{\alpha}, \alpha \in \mathbb{R}$}

As a last example, we consider for $W\left(x_{0}\right)$ the Gegenbauer weight function. The generalized axial CK-extension now takes a slightly different form, namely

$$
f\left(x_{0}, \underline{x}\right)=\sum_{s=0}^{\infty} c_{s, k} \underline{x}^{s} G_{s, k}^{\alpha}\left(x_{0}\right)\left(1-x_{0}^{2}\right)^{\alpha-s} P_{k}(\underline{x})
$$

with $G_{0, k}^{\alpha}\left(x_{0}\right)=1$ and the coefficients $c_{s, k}$ again satisfying (1). We indeed have that

$$
\lim _{|\underline{x}| \rightarrow 0} \frac{1}{|\underline{x}|^{k}} f\left(x_{0}, \underline{x}\right)=\left(1-x_{0}^{2}\right)^{\alpha} P_{k}(\underline{\omega}) .
$$

First the aim is to determine $G_{s, k}^{\alpha}\left(x_{0}\right)$ and then the series $\sum_{s=0}^{\infty} c_{s, k} \underline{x}^{s} G_{s, k}^{\alpha}(0) P_{k}(\underline{x})$. The monogenicity relation $\left(\partial_{x_{0}}+\partial_{\underline{x}}\right)\left[f\left(x_{0}, \underline{x}\right)\right]=0$, leads to the recurrence relation

$$
G_{s+1, k}^{\alpha}\left(x_{0}\right)=2(\alpha-s) x_{0} G_{s, k}^{\alpha}\left(x_{0}\right)-\left(1-x_{0}^{2}\right)\left(G_{s, k}^{\alpha}\right)^{\prime}\left(x_{0}\right), \quad G_{0, k}^{\alpha}\left(x_{0}\right)=1 .
$$

The results below concerning the polynomials $G_{s, k}^{\alpha}\left(x_{0}\right)$ are already mentioned in [4].

The polynomials of lower degree take the form

$$
\begin{aligned}
& G_{0, k}^{\alpha}\left(x_{0}\right)=1 \\
& G_{1, k}^{\alpha}\left(x_{0}\right)=2 \alpha x_{0} \\
& G_{2, k}^{\alpha}\left(x_{0}\right)=2 \alpha(2 \alpha-1) x_{0}^{2}-2 \alpha \\
& G_{3, k}^{\alpha}\left(x_{0}\right)=4 \alpha(\alpha-1)(2 \alpha-1) x_{0}^{3}-12 \alpha(\alpha-1) x_{0} \\
& \quad \text { etc. }
\end{aligned}
$$


Putting

$$
G_{2 t, k}^{\alpha}\left(x_{0}\right)=\sum_{i=0}^{t} b_{2 i}^{2 t, \alpha} x_{0}^{2 i} \quad \text { and } \quad G_{2 t+1, k}^{\alpha}\left(x_{0}\right)=\sum_{i=0}^{t} b_{2 i+1}^{2 t+1, \alpha} x_{0}^{2 i+1},
$$

we can derive an expression for the coefficients $b_{2 i}^{2 t, \alpha}$ and $b_{2 i+1}^{2 t+1, \alpha}$.

Theorem 2.2 For $0 \leq i \leq t$, one has

$$
\begin{aligned}
b_{2 i}^{2 t, \alpha} & =(-1)^{t} 2^{2 t}(2 t) ! \frac{(\alpha-2 t+1)_{2 t}}{(2 \alpha-4 t+1)_{2 t} \Gamma(\alpha-2 t+1 / 2)}(-1)^{i} \frac{2^{2 i} \Gamma(\alpha+1 / 2-t+i)}{(t-i) !(2 i) !} \\
b_{2 i+1}^{2 t+1, \alpha} & =(-1)^{t} 2^{2 t+1}(2 t+1) ! \frac{(\alpha-2 t)_{2 t+1}}{(2 \alpha-4 t-1)_{2 t+1} \Gamma(\alpha-2 t-1 / 2)}(-1)^{i} \\
& \times \frac{2^{2 i+1} \Gamma(\alpha+1 / 2-t+i)}{(t-i) !(2 i+1) !}
\end{aligned}
$$

with $(\alpha)_{\ell}=\alpha(\alpha+1) \ldots(\alpha+\ell-1)=\frac{\Gamma(\alpha+\ell)}{\Gamma(\alpha)}$ Pochhammer's symbol.

Proof. This result is proved by means of induction on the degree of the polynomial and using the recurrence relation (6).

Using the above theorem, we can write $G_{t, k}^{\alpha}\left(x_{0}\right)$ in terms of the classical Gegenbauer polynomials on the real line.

\section{Corollary 2.1 One has}

$$
\begin{aligned}
G_{2 t, k}^{\alpha}\left(x_{0}\right) & =2^{2 t}(2 t) ! \frac{(\alpha-2 t+1)_{2 t}}{(2 \alpha-4 t+1)_{2 t}} C_{2 t}^{\alpha-2 t+1 / 2}\left(x_{0}\right) \\
G_{2 t+1, k}^{\alpha}\left(x_{0}\right) & =2^{2 t+1}(2 t+1) ! \frac{(\alpha-2 t)_{2 t+1}}{(2 \alpha-4 t-1)_{2 t+1}} C_{2 t+1}^{\alpha-2 t-1 / 2}\left(x_{0}\right)
\end{aligned}
$$

with $C_{n}^{\lambda}\left(x_{0}\right)$ the classical Gegenbauer polynomial on the real line given explicitly by (see e.g. [10], p. 219)

$$
C_{n}^{\lambda}\left(x_{0}\right)=\frac{1}{\Gamma(\lambda)} \sum_{i=0}^{\left\lfloor\frac{n}{2}\right\rfloor} \frac{(-1)^{i} \Gamma(\lambda+n-i)}{i !(n-2 i) !}\left(2 x_{0}\right)^{n-2 i}, \quad \lambda>0 .
$$

Proof. This follows immediately from Theorem 2.2 and the explicit expression (7). 
From the above it follows that $G_{2 t+1, k}^{\alpha}(0)=0$ and

$$
G_{2 t, k}^{\alpha}(0)=b_{0}^{2 t, \alpha}=(-1)^{t} 2^{2 t} \frac{(2 t) !}{t !} \frac{\Gamma(\alpha+1) \Gamma(2 \alpha-4 t+1) \Gamma(\alpha+1 / 2-t)}{\Gamma(\alpha-2 t+1) \Gamma(2 \alpha-2 t+1) \Gamma(\alpha-2 t+1 / 2)} .
$$

In view of (see e.g. [10], p. 3):

$$
\Gamma(2 z)=\pi^{-1 / 2} 2^{2 z-1} \Gamma(z) \Gamma\left(z+\frac{1}{2}\right)
$$

the expression for $G_{2 t, k}^{\alpha}(0)$ can be simplified to

$$
G_{2 t, k}^{\alpha}(0)=(-1)^{t} \frac{(2 t) !}{t !} \frac{\Gamma(\alpha+1)}{\Gamma(\alpha-t+1)}
$$

We thus obtain

$$
\begin{aligned}
\sum_{s=0}^{\infty} c_{s, k} \underline{x}^{s} G_{s, k}^{\alpha}(0) P_{k}(\underline{x}) & =\sum_{\ell=0}^{\infty} c_{2 \ell, k} \underline{x}^{2 \ell} G_{2 \ell, k}^{\alpha}(0) P_{k}(\underline{x}) \\
& =\sum_{\ell=0}^{\infty} \frac{\Gamma\left(\frac{m}{2}+k\right)}{\Gamma\left(\frac{m}{2}+k+\ell\right) 2^{2 \ell} \ell !}|\underline{x}|^{2 \ell} \frac{(2 \ell) !}{\ell !} \frac{\Gamma(\alpha+1)}{\Gamma(\alpha-\ell+1)} P_{k}(\underline{x}) .
\end{aligned}
$$

Taking into account (5) and (see e.g. [10], p. 2):

$$
\Gamma(\alpha+1-\ell)=(-1)^{\ell} \Gamma(\alpha+1) \frac{\Gamma(-\alpha)}{\Gamma(\ell-\alpha)},
$$

expression (9) becomes

$$
\begin{aligned}
\sum_{s=0}^{\infty} c_{s, k} \underline{x}^{s} G_{s, k}^{\alpha}(0) P_{k}(\underline{x}) & =\sum_{\ell=0}^{\infty} \frac{\Gamma\left(\frac{m}{2}+k\right)}{\Gamma\left(\frac{m}{2}+k+\ell\right)} \frac{\Gamma\left(\ell+\frac{1}{2}\right)}{\Gamma\left(\frac{1}{2}\right)} \frac{\Gamma(\ell-\alpha)}{\Gamma(-\alpha)} \frac{\left(-|\underline{x}|^{2}\right)^{\ell}}{\ell !} P_{k}(\underline{x}) \\
& ={ }_{2} F_{1}\left(1 / 2,-\alpha ; m / 2+k ;-|\underline{x}|^{2}\right) P_{k}(\underline{x})
\end{aligned}
$$

with

$$
{ }_{2} F_{1}(a, b ; c ; z)=\sum_{\ell=0}^{\infty} \frac{\Gamma(a+\ell)}{\Gamma(a)} \frac{\Gamma(b+\ell)}{\Gamma(b)} \frac{\Gamma(c)}{\Gamma(c+\ell)} \frac{z^{\ell}}{\ell !}
$$

the hypergeometric function. 


\section{Generalized Cauchy-Kowalevski extensions: the biaxial framework}

In this section we consider functions of two vector variables $\underline{x}=\sum_{j=1}^{p} x_{j} e_{j}$ and $\underline{y}=$ $\sum_{j=1}^{q} y_{j} e_{p+j}$. The sum $\underline{x}+\underline{y}$ represents a vector variable in $\mathbb{R}^{p+q}=\mathbb{R}^{m}$ equipped with the orthonormal basis $\left(e_{1}, \ldots, e_{p}, e_{p+1}, \ldots, e_{p+q}\right)$. The corresponding Dirac operators take the form $\partial_{\underline{x}}=\sum_{j=1}^{p} \partial_{x_{j}} e_{j}$ and $\partial_{\underline{y}}=\sum_{j=1}^{q} \partial_{y_{j}} e_{p+j}$. Note that the vector variables and Dirac operators anti-commute, i.e. $\underline{x} \underline{y}=-\underline{y} \underline{x}$ and $\partial_{\underline{x}} \partial_{\underline{y}}=-\partial_{\underline{y}} \partial_{\underline{x}}$ and that the vector variables are orthogonal, i.e. $\langle\underline{x}, \underline{y}\rangle=0$.

In the following, $P_{k^{\prime}}(\underline{x})$ and $P_{\ell^{\prime}}(y)$ represent solid inner spherical monogenics of degree $k^{\prime}$ in the variable $\underline{x}$ and of degree $\ell^{\prime}$ in the variable $\underline{y}$. This means that $P_{k^{\prime}}(\underline{x})$ and $P_{\ell^{\prime}}(\underline{y})$ are homogeneous polynomials taking values in the Clifford algebra spanned by respectively $\left\{e_{1}, \ldots, e_{p}\right\}$ and $\left\{e_{p+1}, \ldots, e_{p+q}\right\}$; they satisfy the relations $\partial_{\underline{x}}\left[P_{k^{\prime}}(\underline{x})\right]=0, P_{k^{\prime}}(\underline{x})=$ $t^{k^{\prime}} P_{k^{\prime}}(\underline{x})$ and $\partial_{\underline{y}}\left[P_{\ell^{\prime}}(\underline{y})\right]=0, P_{\ell^{\prime}}(t \underline{y})=t^{\ell^{\prime}} P_{\ell^{\prime}}(\underline{y})$. We also assume $P_{k^{\prime}}(\underline{x})$ and $P_{\ell^{\prime}}(\underline{y})$ to be even Clifford algebra-valued, which implies that $P_{k^{\prime}}(\underline{x})$ and $P_{\ell^{\prime}}(\underline{y})$ commute.

Let us first introduce the concept of generalized CK-extensions in this biaxial framework. Consider an $S O(q)$-invariant domain $\widetilde{\Omega} \subset \mathbb{R}^{m}$ and assume that the intersections of $\widetilde{\Omega}$ with all subspaces parallel to $\mathbb{R}^{q}$ are convex. Putting $\Omega=\widetilde{\Omega} \cap \mathbb{R}^{p}$, the following holds (see [3]).

Theorem 3.1 Let $P_{\ell^{\prime}}(\underline{y}) \in \mathcal{M}_{\ell^{\prime}}(q)$ be fixed and let $W_{0}(\underline{x})$ be a fixed analytic function in $\Omega$. Then there exists a unique sequence $\left(W_{s}(\underline{x})\right)_{s>0}$ of analytic functions such that the series

$$
f(\underline{x}, \underline{y})=\sum_{s=0}^{\infty} \underline{y}^{s} P_{\ell^{\prime}}(\underline{y}) W_{s}(\underline{x})
$$

is convergent in a neighbourhood $U \subset \mathbb{R}^{m}$ of the domain $\Omega$ and its sum $f$ is monogenic in $U$. The function $W_{0}(\underline{x})$ is determined by the relation

$$
P_{\ell^{\prime}}(\underline{\xi}) W_{0}(\underline{x})=\lim _{|\underline{y}| \rightarrow 0} \frac{1}{|\underline{y}|^{\ell^{\prime}}} f(\underline{x}, \underline{y}), \quad \underline{y}=|\underline{y}| \underline{\xi} .
$$

The function $f(\underline{x}, \underline{y})$ is the so-called generalized biaxial CK-extension of $W_{0}(\underline{x})$. 
3.1 First example: $W_{0}(\underline{x})=e^{-|\underline{x}|^{2} / 2} P_{k^{\prime}}(\underline{x})$

Given

$$
f(\underline{x}, \underline{y})=\sum_{s=0}^{\infty} c_{s, \ell^{\prime}} \underline{y}^{s} P_{\ell^{\prime}}(\underline{y}) H_{s, k^{\prime}}(\underline{x}) e^{-|\underline{x}|^{2} / 2} P_{k^{\prime}}(\underline{x})
$$

with $H_{0, k^{\prime}}(\underline{x})=1$,

$$
\partial_{\underline{y}}\left[c_{s, \ell^{\prime}} \underline{y}^{s} P_{\ell^{\prime}}(\underline{y})\right]=c_{s-1, \ell^{\prime}} \underline{y}^{s-1} P_{\ell^{\prime}}(\underline{y}), \quad c_{0, \ell^{\prime}}=1
$$

and $\left(\partial_{\underline{x}}+\partial_{\underline{y}}\right)[f(\underline{x}, \underline{y})]=0$, the question is to determine

$$
\lim _{|\underline{x}| \rightarrow 0} \frac{1}{|\underline{x}|^{k^{\prime}}} f(\underline{x}, \underline{y})=\sum_{s=0}^{\infty} c_{s, \ell^{\prime}} \underline{y}^{s} P_{\ell^{\prime}}(\underline{y}) H_{s, k^{\prime}}(\underline{0}) P_{k^{\prime}}(\underline{\omega})
$$

In a similar way as in the previous section, we obtain that the coefficients $c_{s, \ell^{\prime}}$ are

$$
c_{2 \ell, \ell^{\prime}}=\frac{\Gamma\left(\frac{q}{2}+\ell^{\prime}\right)}{\Gamma\left(\frac{q}{2}+\ell^{\prime}+\ell\right) 2^{2 \ell} \ell !} \quad \text { and } \quad c_{2 \ell+1, \ell^{\prime}}=-\frac{\Gamma\left(\frac{q}{2}+\ell^{\prime}\right)}{\Gamma\left(\frac{q}{2}+\ell^{\prime}+\ell+1\right) 2^{2 \ell+1} \ell !} .
$$

From the monogenicity of $f$, we obtain consecutively

$$
\begin{aligned}
& \left(\partial_{\underline{x}}+\partial_{\underline{y}}\right)\left[\sum_{s=0}^{\infty} c_{s, \ell^{\prime}} \underline{y}^{s} P_{\ell^{\prime}}(\underline{y}) H_{s, k^{\prime}}(\underline{x}) e^{-|\underline{x}|^{2} / 2} P_{k^{\prime}}(\underline{x})\right]=0 \\
& \Longleftrightarrow \sum_{s=0}^{\infty} c_{s, \ell^{\prime}}(-1)^{s} \underline{y}^{s} P_{\ell^{\prime}}(\underline{y})\left(-\underline{x} H_{s, k^{\prime}}(\underline{x}) P_{k^{\prime}}(\underline{x})+\partial_{\underline{x}}\left[H_{s, k^{\prime}}(\underline{x}) P_{k^{\prime}}(\underline{x})\right]\right) e^{-|\underline{x}|^{2} / 2} \\
& +\sum_{s=0}^{\infty} c_{s, \ell^{\prime}} \underline{y}^{s} P_{\ell^{\prime}}(\underline{y}) H_{s+1, k^{\prime}}(\underline{x}) e^{-|\underline{x}|^{2} / 2} P_{k^{\prime}}(\underline{x})=0 .
\end{aligned}
$$

The functions $H_{s, k^{\prime}}(\underline{x})$ thus satisfy the recurrence relation

$$
H_{s+1, k^{\prime}}(\underline{x}) P_{k^{\prime}}(\underline{x})=(-1)^{s}\left(\underline{x}-\partial_{\underline{x}}\right)\left[H_{s, k^{\prime}}(\underline{x}) P_{k^{\prime}}(\underline{x})\right], \quad H_{0, k^{\prime}}(\underline{x})=1,
$$

from which we observe that

$$
H_{2 \ell, k^{\prime}}(\underline{x})=(-1)^{\ell} H_{2 \ell, k^{\prime}}^{G C H}(\underline{x}) \quad \text { and } \quad H_{2 \ell+1, k^{\prime}}(\underline{x})=(-1)^{\ell} H_{2 \ell+1, k^{\prime}}^{G C H}(\underline{x})
$$

with $H_{s, k^{\prime}}^{G C H}(\underline{x})$ the so-called generalized Clifford-Hermite polynomials introduced by Sommen in [15]. Taking into account the expression of these polynomials in terms of the 
generalized Laguerre polynomials on the real line

$$
H_{2 \ell, k^{\prime}}^{G C H}(\underline{x})=2^{\ell} \ell ! L_{\ell}^{p / 2+k^{\prime}-1}\left(\frac{|\underline{x}|^{2}}{2}\right) \quad \text { and } \quad H_{2 \ell+1, k^{\prime}}^{G C H}(\underline{x})=2^{\ell} \ell ! L_{\ell}^{p / 2+k^{\prime}}\left(\frac{|\underline{x}|^{2}}{2}\right) \underline{x},
$$

and the relation (see e.g. [10], p. 240): $L_{n}^{\alpha}(0)=\frac{(\alpha+1)_{n}}{n !}$, we have that

$$
H_{2 \ell, k^{\prime}}(\underline{0})=(-1)^{\ell} 2^{\ell} \frac{\Gamma\left(\frac{p}{2}+k^{\prime}+\ell\right)}{\Gamma\left(\frac{p}{2}+k^{\prime}\right)} \quad \text { and } \quad H_{2 \ell+1, k^{\prime}}(\underline{0})=0 .
$$

This finally yields

$$
\begin{aligned}
\lim _{|\underline{x}| \rightarrow 0} \frac{1}{|\underline{x}|^{k^{\prime}}} f(\underline{x}, \underline{y}) & =\sum_{\ell=0}^{\infty} c_{2 \ell, \ell^{\prime}} \underline{y}^{2 \ell} P_{\ell^{\prime}}(\underline{y}) H_{2 \ell, k^{\prime}}(\underline{0}) P_{k^{\prime}}(\underline{\omega}) \\
& =\frac{\Gamma\left(\frac{q}{2}+\ell^{\prime}\right)}{\Gamma\left(\frac{p}{2}+k^{\prime}\right)} \sum_{\ell=0}^{\infty} \frac{\Gamma\left(\frac{p}{2}+k^{\prime}+\ell\right)}{\Gamma\left(\frac{q}{2}+\ell^{\prime}+\ell\right)} \frac{1}{\ell !}\left(\frac{|\underline{y}|^{2}}{2}\right)^{\ell} P_{\ell^{\prime}}(\underline{y}) P_{k^{\prime}}(\underline{\omega}) \\
& ={ }_{1} F_{1}\left(p / 2+k^{\prime} ; q / 2+\ell^{\prime} ; \frac{|\underline{y}|^{2}}{2}\right) P_{\ell^{\prime}}(\underline{y}) P_{k^{\prime}}(\underline{\omega}) .
\end{aligned}
$$

3.2 Second example: $W_{0}(\underline{x})=\left(\widetilde{J}_{k^{\prime}+p / 2-1}(|\underline{x}|)+\underline{x} \widetilde{J}_{k^{\prime}+p / 2}(|\underline{x}|)\right) P_{k^{\prime}}(\underline{x})$

In this subsection we determine the generalized biaxial CK-extension of the Clifford-Bessel function without exponential factor, introduced by Sommen in [15]. We now put

$$
f(\underline{x}, \underline{y})=\sum_{s=0}^{\infty} c_{s, \ell^{\prime}} \underline{y}^{s} P_{\ell^{\prime}}(\underline{y}) \partial_{\underline{x}}^{s}\left[\left(\widetilde{J}_{k^{\prime}+p / 2-1}(|\underline{x}|)+\underline{x} \widetilde{J}_{k^{\prime}+p / 2}(|\underline{x}|)\right) P_{k^{\prime}}(\underline{x})\right]
$$

where the coefficients $c_{s, \ell^{\prime}}$ are taken such that $\sum_{s=0}^{\infty} c_{s, \ell^{\prime}} \underline{y}^{s} P_{\ell^{\prime}}(\underline{y}) \partial_{\underline{x}}^{s}$ is monogenic, i.e. $\left(\partial_{\underline{x}}+\partial_{\underline{y}}\right)\left[\sum_{s=0}^{\infty} c_{s, \ell^{\prime}} \underline{y}^{s} P_{\ell^{\prime}}(\underline{y}) \partial_{\underline{x}}^{s}\right]=0$. This leads to the equation

$$
c_{s, \ell^{\prime}} \partial_{\underline{y}}\left[\underline{y}^{s} P_{\ell^{\prime}}(\underline{y})\right]=(-1)^{s} c_{s-1, \ell^{\prime}} \underline{y}^{s-1} P_{\ell^{\prime}}(\underline{y}) .
$$

As $\lim _{|\underline{y}| \rightarrow 0} \frac{1}{\mid \underline{|y|^{\ell^{\prime}}}} f(\underline{x}, \underline{y})=W_{0}(\underline{x}) P_{\ell^{\prime}}(\underline{\xi})$, we also have that $c_{0, \ell^{\prime}}=1$. By means of induction, one can prove that in this case the coefficients $c_{s, \ell^{\prime}}$ take the form

$$
c_{2 \ell, \ell^{\prime}}=\frac{(-1)^{\ell} \Gamma\left(\frac{q}{2}+\ell^{\prime}\right)}{\Gamma\left(\frac{q}{2}+\ell^{\prime}+\ell\right) 2^{2 \ell} \ell !} \text { and } c_{2 \ell+1, \ell^{\prime}}=\frac{(-1)^{\ell} \Gamma\left(\frac{q}{2}+\ell^{\prime}\right)}{\Gamma\left(\frac{q}{2}+\ell^{\prime}+\ell+1\right) 2^{2 \ell+1} \ell !} .
$$


The question is to determine $\lim _{|\underline{x}| \rightarrow 0} \frac{1}{|\underline{x}|^{k^{\prime}}} f(\underline{x}, \underline{y})$. Hereto we start with the following result.

Theorem 3.2 One has

$$
\partial_{\underline{x}}^{s}\left[\left(\widetilde{J}_{k^{\prime}+p / 2-1}(|\underline{x}|)+\underline{x} \widetilde{J}_{k^{\prime}+p / 2}(|\underline{x}|)\right) P_{k^{\prime}}(\underline{x})\right]=(-1)^{s}\left(\widetilde{J}_{k^{\prime}+p / 2-1}(|\underline{x}|)+\underline{x} \widetilde{J}_{k^{\prime}+p / 2}(|\underline{x}|)\right) P_{k^{\prime}}(\underline{x}) .
$$

Proof. By means of the series expansion (4) for the Bessel function we have that

$$
\begin{aligned}
\left(\widetilde{J}_{k^{\prime}+p / 2-1}(|\underline{x}|)\right. & \left.+\underline{x}_{k^{\prime}+p / 2}(|\underline{x}|)\right) P_{k^{\prime}}(\underline{x}) \\
& =2^{1-p / 2-k^{\prime}} \sum_{\ell=0}^{\infty} \frac{\underline{x}^{2 \ell} P_{k^{\prime}}(\underline{x})}{\ell ! \Gamma\left(\frac{p}{2}+k^{\prime}+\ell\right) 2^{2 \ell}}+2^{-p / 2-k^{\prime}} \sum_{\ell=0}^{\infty} \frac{\underline{x}^{2 \ell+1} P_{k^{\prime}}(\underline{x})}{\ell ! \Gamma\left(\frac{p}{2}+k^{\prime}+\ell+1\right) 2^{2 \ell}} .
\end{aligned}
$$

Using the fundamental formula (2) and executing the substitution $\ell^{\prime}=\ell-1$ in the first series, we have consecutively

$$
\begin{aligned}
& \partial_{\underline{x}}\left[\left(\widetilde{J}_{k^{\prime}+p / 2-1}(|\underline{x}|)+\underline{x} \widetilde{J}_{k^{\prime}+p / 2}(|\underline{x}|)\right) P_{k^{\prime}}(\underline{x})\right] \\
& =-2^{1-p / 2-k^{\prime}} \sum_{\ell=1}^{\infty} \frac{\underline{x}^{2 \ell-1} P_{k^{\prime}}(\underline{x})}{(\ell-1) ! \Gamma\left(\frac{p}{2}+k^{\prime}+\ell\right) 2^{2 \ell-1}}-2^{-p / 2-k^{\prime}} \sum_{\ell=0}^{\infty} \frac{\left(2 \ell+p+2 k^{\prime}\right) \underline{x}^{2 \ell} P_{k^{\prime}}(\underline{x})}{\ell ! \Gamma\left(\frac{p}{2}+k^{\prime}+\ell+1\right) 2^{2 \ell}} \\
& =-2^{-p / 2-k^{\prime}} \sum_{\ell^{\prime}=0}^{\infty} \frac{\underline{x}^{2 \ell^{\prime}+1} P_{k^{\prime}}(\underline{x})}{\ell^{\prime} ! \Gamma\left(\frac{p}{2}+k^{\prime}+\ell^{\prime}+1\right) 2^{2 \ell^{\prime}}}-2^{-p / 2-k^{\prime}+1} \sum_{\ell=0}^{\infty} \frac{\underline{x}^{2 \ell} P_{k^{\prime}}(\underline{x})}{\ell ! \Gamma\left(\frac{p}{2}+k^{\prime}+\ell\right) 2^{2 \ell}} \\
& =-\left(\widetilde{J}_{k^{\prime}+p / 2-1}(|\underline{x}|)+\underline{x} \widetilde{J}_{k^{\prime}+p / 2}(|\underline{x}|)\right) P_{k^{\prime}}(\underline{x}),
\end{aligned}
$$

from which the desired result follows.

We thus have that

$$
f(\underline{x}, \underline{y})=\sum_{s=0}^{\infty} c_{s, \ell^{\prime}} \underline{y}^{s} P_{\ell^{\prime}}(\underline{y})(-1)^{s}\left(\widetilde{J}_{k^{\prime}+p / 2-1}(|\underline{x}|)+\underline{x} \widetilde{J}_{k^{\prime}+p / 2}(|\underline{x}|)\right) P_{k^{\prime}}(\underline{x})
$$


which leads to

$$
\begin{aligned}
\lim _{|\underline{x}| \rightarrow 0} \frac{1}{|\underline{x}|^{k^{\prime}}} f(\underline{x}, \underline{y}) & =\sum_{s=0}^{\infty} c_{s, \ell^{\prime}} \underline{y}^{s} P_{\ell^{\prime}}(\underline{y})(-1)^{s} \frac{2^{1-p / 2-k^{\prime}}}{\Gamma\left(\frac{p}{2}+k^{\prime}\right)} P_{k^{\prime}}(\underline{\omega}) \\
& =\frac{2^{1-p / 2-k^{\prime}}}{\Gamma\left(\frac{p}{2}+k^{\prime}\right)}\left(\sum_{\ell=0}^{\infty} c_{2 \ell, \ell^{\prime}} \underline{y}^{2 \ell}-\sum_{\ell=0}^{\infty} c_{2 \ell+1, \ell^{\prime}} \underline{y}^{2 \ell+1}\right) P_{\ell^{\prime}}(\underline{y}) P_{k^{\prime}}(\underline{\omega}) \\
& =2^{1-p / 2-k^{\prime}} \frac{\Gamma\left(\frac{q}{2}+\ell^{\prime}\right)}{\Gamma\left(\frac{p}{2}+k^{\prime}\right)}\left(\sum_{\ell=0}^{\infty} \frac{|\underline{y}|^{2 \ell}}{\Gamma\left(\frac{q}{2}+\ell^{\prime}+\ell\right) 2^{2 \ell} \ell !}\right. \\
& \left.-\underline{y} \sum_{\ell=0}^{\infty} \frac{|y|^{2 \ell}}{\Gamma\left(\frac{q}{2}+\ell^{\prime}+\ell+1\right) 2^{2 \ell+1} \ell !}\right) P_{\ell^{\prime}}(\underline{y}) P_{k^{\prime}}(\underline{\omega}) .
\end{aligned}
$$

The above calculations lead to the following result.

Theorem 3.3 One has that

$$
\begin{aligned}
& \lim _{|\underline{x}| \rightarrow 0} \frac{1}{|\underline{x}|^{k^{\prime}}} f(\underline{x}, \underline{y}) \\
& =2^{(q-p) / 2+\ell^{\prime}-k^{\prime}} \frac{\Gamma\left(\frac{q}{2}+\ell^{\prime}\right)}{\Gamma\left(\frac{p}{2}+k^{\prime}\right)} P_{k^{\prime}}(\underline{\omega}) P_{\ell^{\prime}}(\underline{y})\left(\widetilde{J}_{q / 2+\ell^{\prime}-1}(i|\underline{y}|)-\widetilde{J}_{q / 2+\ell^{\prime}}(i|\underline{y}|) \underline{y}\right) \\
& =2^{(q-p) / 2+\ell^{\prime}-k^{\prime}} \frac{\Gamma\left(\frac{q}{2}+\ell^{\prime}\right)}{\Gamma\left(\frac{p}{2}+k^{\prime}\right)} P_{k^{\prime}}(\underline{\omega}) P_{\ell^{\prime}}(\underline{y})|\underline{y}|^{1-q / 2-\ell^{\prime}}\left(I_{q / 2+\ell^{\prime}-1}(|\underline{y}|)-\frac{y}{|\underline{y}|} I_{q / 2+\ell^{\prime}}(|\underline{y}|)\right)
\end{aligned}
$$

with $I_{\nu}(z)$ the modified Bessel function of the first kind.

Proof. This theorem is proved using the series expansion (4) and the relations (see e.g. [10])

$$
\widetilde{J}_{\nu}(z)=z^{-\nu} J_{\nu}(z), \quad J_{\nu}(i z)=i^{\nu} I_{\nu}(z)
$$

Remark 3.1 During the above calculations we have also obtained a closed form for the 
full generalized biaxial CK-extension:

$$
\begin{aligned}
& f(\underline{x}, \underline{y})=2^{q / 2+\ell^{\prime}-1} \Gamma\left(\frac{q}{2}+\ell^{\prime}\right) P_{\ell^{\prime}}(\underline{y})\left(\widetilde{J}_{q / 2+\ell^{\prime}-1}(i|\underline{y}|)-\widetilde{J}_{q / 2+\ell^{\prime}}(i|\underline{y}|) \underline{y}\right) \\
& \times\left(\widetilde{J}_{p / 2+k^{\prime}-1}(|\underline{x}|)+\underline{x} \widetilde{J}_{p / 2+k^{\prime}}(|\underline{x}|)\right) P_{k^{\prime}}(\underline{x}) \\
& =2^{q / 2+\ell^{\prime}-1} \Gamma\left(\frac{q}{2}+\ell^{\prime}\right) P_{\ell^{\prime}}(\underline{y})|\underline{y}|^{1-q / 2-\ell^{\prime}}\left(I_{q / 2+\ell^{\prime}-1}(|\underline{y}|)-\frac{\underline{y}}{|\underline{y}|} I_{q / 2+\ell^{\prime}}(|\underline{y}|)\right) \\
& \times|\underline{x}|^{1-p / 2-k^{\prime}}\left(J_{p / 2+k^{\prime}-1}(|\underline{x}|)+\frac{\underline{x}}{|\underline{x}|} J_{p / 2+k^{\prime}}(|\underline{x}|)\right) P_{k^{\prime}}(\underline{x}) .
\end{aligned}
$$

Note that from the monogenicity of $f$ it follows that the even Clifford algebra-valued part:

$$
\begin{aligned}
{[f(\underline{x}, \underline{y})]_{\mathrm{e}}=2^{q / 2+\ell^{\prime}-1} \Gamma\left(\frac{q}{2}+\ell^{\prime}\right)\left(\widetilde{J}_{p / 2+k^{\prime}-1}(|\underline{x}|) \widetilde{J}_{q / 2+\ell^{\prime}-1}(i|\underline{y}|)\right.} & \\
& \left.+\underline{x} \underline{y} \widetilde{J}_{k^{\prime}+p / 2}(|\underline{x}|) \widetilde{J}_{q / 2+\ell^{\prime}}(i|\underline{y}|)\right) P_{k^{\prime}}(\underline{x}) P_{\ell^{\prime}}(\underline{y})
\end{aligned}
$$

and the odd Clifford algebra-valued part

$$
\begin{aligned}
{[f(\underline{x}, \underline{y})]_{\mathrm{o}}=2^{q / 2+\ell^{\prime}-1} \Gamma\left(\frac{q}{2}+\ell^{\prime}\right)\left(\widetilde{J}_{p / 2+k^{\prime}}(|\underline{x}|) \widetilde{J}_{q / 2+\ell^{\prime}-1}(i|\underline{y}|) \underline{x}\right.} \\
\\
\left.\quad-\widetilde{J}_{k^{\prime}+p / 2-1}(|\underline{x}|) \widetilde{J}_{q / 2+\ell^{\prime}}(i|\underline{y}|) \underline{y}\right) P_{k^{\prime}}(\underline{x}) P_{\ell^{\prime}}(\underline{y})
\end{aligned}
$$

are also monogenic.

3.3 Third example: $W_{0}(\underline{x})={ }_{1} F_{1}\left(a ; c ;-\frac{|\underline{x}|^{2}}{2}\right) P_{k^{\prime}}(\underline{x}), a, c \in \mathbb{R}$

Similar to the previous subsection, we start from the following expression for the generalized biaxial CK-extension

$$
f(\underline{x}, \underline{y})=\sum_{s=0}^{\infty} c_{s, \ell^{\prime}} \underline{y}^{s} P_{\ell^{\prime}}(\underline{y}) \partial_{\underline{x}}^{s}\left[{ }_{1} F_{1}\left(a ; c ;-\frac{|\underline{x}|^{2}}{2}\right) P_{k^{\prime}}(\underline{x})\right]
$$

where the coefficients $c_{s, \ell^{\prime}}$ take the form (10).

We start with calculating the derivatives of $W_{0}(\underline{x})$. 
Proposition 3.1 For $n \in \mathbb{N}$ one has

$$
\begin{aligned}
& \partial_{\underline{x}}^{2 n}\left[{ }_{1} F_{1}\left(a ; c ;-\frac{|\underline{x}|^{2}}{2}\right) P_{k^{\prime}}(\underline{x})\right]=\sum_{i=0}^{n} \frac{(a)_{2 n-i}}{(c)_{2 n-i}} 2^{i}\left(\frac{p}{2}+k^{\prime}+n-i\right)_{i}\left(\begin{array}{c}
n \\
i
\end{array}\right) \\
& \times{ }_{1} F_{1}\left(a+2 n-i ; c+2 n-i ;-\frac{|\underline{x}|^{2}}{2}\right)(-\underline{x})^{2 n-2 i} P_{k^{\prime}}(\underline{x}) \\
& \partial_{\underline{x}}^{2 n+1}\left[{ }_{1} F_{1}\left(a ; c ;-\frac{|\underline{x}|^{2}}{2}\right) P_{k^{\prime}}(\underline{x})\right]=\sum_{i=0}^{n} \frac{(a)_{2 n+1-i}}{(c)_{2 n+1-i}} 2^{i}\left(\frac{p}{2}+k^{\prime}+n-i+1\right)_{i}\left(\begin{array}{c}
n \\
i
\end{array}\right) \\
& \times{ }_{1} F_{1}\left(a+2 n+1-i ; c+2 n+1-i ;-\frac{|\underline{x}|^{2}}{2}\right)(-\underline{x})^{2 n+1-2 i} P_{k^{\prime}}(\underline{x}) .
\end{aligned}
$$

Proof. This result is proved by induction on $n$ using the relations

$$
\partial_{\underline{x}}\left[{ }_{1} F_{1}\left(\alpha ; \beta ;-\frac{|\underline{x}|^{2}}{2}\right)\right]=\frac{\alpha}{\beta}{ }_{1} F_{1}\left(\alpha+1 ; \beta+1 ;-\frac{|\underline{x}|^{2}}{2}\right)(-\underline{x})
$$

and

$$
\partial_{\underline{x}}\left[(-\underline{x})^{\ell} P_{k^{\prime}}(\underline{x})\right]= \begin{cases}\ell(-\underline{x})^{\ell-1} P_{k^{\prime}}(\underline{x}) & \text { for } \ell \text { even } \\ \left(\ell+p-1+2 k^{\prime}\right)(-\underline{x})^{\ell-1} P_{k^{\prime}}(\underline{x}) & \text { for } \ell \text { odd }\end{cases}
$$

The differential equations in Proposition 3.1 lead to

$$
\begin{gathered}
\left(\frac{1}{|\underline{x}|^{k^{\prime}}} \partial_{\underline{x}}^{2 n}\left[{ }_{1} F_{1}\left(a ; c ;-\frac{|\underline{x}|^{2}}{2}\right) P_{k^{\prime}}(\underline{x})\right]\right)_{\mid \underline{x}=\underline{0}}=\frac{(a)_{n}}{(c)_{n}} 2^{n}\left(\frac{p}{2}+k^{\prime}\right)_{n} P_{k^{\prime}}(\underline{\omega}) \\
\left(\frac{1}{|\underline{x}|^{k^{\prime}}} \partial_{\underline{x}}^{2 n+1}\left[{ }_{1} F_{1}\left(a ; c ;-\frac{|\underline{x}|^{2}}{2}\right) P_{k^{\prime}}(\underline{x})\right]\right)_{\mid \underline{x}=\underline{0}}=0 .
\end{gathered}
$$

Hence, we obtain the following boundary value:

$$
\begin{aligned}
\lim _{|\underline{x}| \rightarrow 0} \frac{1}{|\underline{x}|^{k^{\prime}}} f(\underline{x}, \underline{y}) & =\sum_{n=0}^{\infty} c_{2 n, \ell^{\prime}} \underline{y}^{2 n} P_{\ell^{\prime}}(\underline{y}) \frac{(a)_{n}}{(c)_{n}} 2^{n}\left(\frac{p}{2}+k^{\prime}\right)_{n} P_{k^{\prime}}(\underline{\omega}) \\
& =\sum_{n=0}^{\infty} \frac{(a)_{n}\left(\frac{p}{2}+k^{\prime}\right)_{n}}{(c)_{n}\left(\frac{q}{2}+\ell^{\prime}\right)_{n}} \frac{1}{n !}\left(\frac{|y|^{2}}{2}\right)^{n} P_{\ell^{\prime}}(\underline{y}) P_{k^{\prime}}(\underline{\omega}) \\
& ={ }_{2} F_{2}\left(a, \frac{p}{2}+k^{\prime} ; c, \frac{q}{2}+\ell^{\prime} ; \frac{|\underline{y}|^{2}}{2}\right) P_{\ell^{\prime}}(\underline{y}) P_{k^{\prime}}(\underline{\omega}) .
\end{aligned}
$$


Remark 3.2 The above calculations can be generalized to:

$$
W_{0}(\underline{x})={ }_{k} F_{\ell}\left(a_{1}, \ldots, a_{k} ; c_{1}, \ldots, c_{\ell} ;-\frac{|\underline{x}|^{2}}{2}\right) P_{k^{\prime}}(\underline{x})
$$

with $a_{i} \in \mathbb{R}(i=1, \ldots, k), c_{j} \in \mathbb{R}(j=1, \ldots, \ell)$. In a similar way as above, we obtain

$$
\lim _{|\underline{x}| \rightarrow 0} \frac{1}{|\underline{x}|^{k^{\prime}}} f(\underline{x}, \underline{y})={ }_{k+1} F_{\ell+1}\left(\frac{p}{2}+k^{\prime}, a_{1}, \ldots, a_{k} ; \frac{q}{2}+\ell^{\prime}, c_{1}, \ldots, c_{\ell} ; \frac{|\underline{y}|^{2}}{2}\right) P_{\ell^{\prime}}(\underline{y}) P_{k^{\prime}}(\underline{\omega}) .
$$

\section{Monogenic plane waves}

\subsection{General method}

For this method we refer to [16].

In what follows, we will frequently use the following Funk-Hecke theorem (see for e.g. [7]).

Theorem 4.1 (Funk-Hecke theorem) Let $S_{k}$ be a spherical harmonic of degree $k$, then

$$
\int_{S^{m-1}} f(\langle\underline{\omega}, \underline{\eta}\rangle) S_{k}(\underline{\omega}) d S(\underline{\omega})=A_{m-1} S_{k}(\underline{\eta}) \int_{-1}^{1} f(t)\left(1-t^{2}\right)^{(m-3) / 2} P_{k, m}(t) d t
$$

where $P_{k, m}(t)$ denotes the Legendre polynomial of degree $k$ in $\mathbb{R}^{m}$, expressed as follows in terms of the Gegenbauer polynomials

$$
P_{k, m}(t)=\frac{k !(m-3) !}{(k+m-3) !} C_{k}^{(m-2) / 2}(t),
$$

and $A_{m-1}=\frac{2 \pi^{(m-1) / 2}}{\Gamma\left(\frac{m-1}{2}\right)}$ the area of the unit sphere $S^{m-2}$ in $\mathbb{R}^{m-1}$.

\subsubsection{The axial case}

Let $h(z)$ be a holomorphic function in $z \in \mathbb{C}$. Consider $h\left(x_{0}+i\langle\underline{x}, \underline{t}\rangle\right)(1-i \underline{t})$ with $x_{0} \in \mathbb{R}$, $\underline{x} \in \mathbb{R}^{m}$ and $\underline{t} \in S^{m-1}$. We have that

$$
\left(\partial_{x_{0}}+\partial_{\underline{x}}\right)\left[h\left(x_{0}+i\langle\underline{x}, \underline{t}\rangle\right)(1-i \underline{t})\right]=h^{\prime}\left(x_{0}+i\langle\underline{x}, \underline{t}\rangle\right)(1+i \underline{t})(1-i \underline{t})=0 .
$$


In the sequel, we consider the following integral:

$$
f\left(x_{0}, \underline{x}\right)=C \int_{S^{m-1}} h\left(x_{0}+i\langle\underline{x}, \underline{t}\rangle\right)(1-i \underline{t}) P_{k}(\underline{t}) d \underline{t}
$$

with $C$ a constant and $P_{k}(\underline{t})$ a fixed inner spherical monogenic of degree $k$. Naturally, we have that $\left(\partial_{x_{0}}+\partial_{\underline{x}}\right)\left[f\left(x_{0}, \underline{x}\right)\right]=0$. Let us now determine $\lim _{|\underline{x}| \rightarrow 0} \frac{1}{|\underline{x}|^{k}} f\left(x_{0}, \underline{x}\right)$. Hereto, we first expand $h\left(x_{0}+i\langle\underline{x}, \underline{t}\rangle\right)$ into a Taylor series:

$$
h\left(x_{0}+i\langle\underline{x}, \underline{t}\rangle\right)=\sum_{\ell=0}^{\infty} \frac{(i\langle\underline{x}, \underline{t}\rangle)^{\ell}}{\ell !} h^{(\ell)}\left(x_{0}\right) .
$$

Hence, we have that

$$
\int_{S^{m-1}} h\left(x_{0}+i\langle\underline{x}, \underline{t}\rangle\right)(1-i \underline{t}) P_{k}(\underline{t}) d \underline{t}=\sum_{\ell=0}^{\infty} \frac{h^{(\ell)}\left(x_{0}\right)}{\ell !} i^{\ell} \int_{S^{m-1}}\langle\underline{x}, \underline{t}\rangle^{\ell}(1-i \underline{t}) P_{k}(\underline{t}) d \underline{t} .
$$

Let us consider the integral over $S^{m-1}$ in the case when $\ell<k$. Application of the FunkHecke theorem yields

$$
\begin{aligned}
& \int_{S^{m-1}}\langle\underline{x}, \underline{t}\rangle^{\ell}(1-i \underline{t}) P_{k}(\underline{t}) d \underline{t} \\
& =\frac{2 \pi^{(m-1) / 2}}{\Gamma\left(\frac{m-1}{2}\right)} r^{\ell}\left(P_{k}(\underline{\omega}) \frac{k !(m-3) !}{(k+m-3) !} \int_{-1}^{1} t^{\ell}\left(1-t^{2}\right)^{(m-3) / 2} C_{k}^{(m-2) / 2}(t) d t\right. \\
& \left.-i \underline{\omega} P_{k}(\underline{\omega}) \frac{(k+1) !(m-3) !}{(k+m-2) !} \int_{-1}^{1} t^{\ell}\left(1-t^{2}\right)^{(m-3) / 2} C_{k+1}^{(m-2) / 2}(t) d t\right),
\end{aligned}
$$

where we have used spherical coordinates $\underline{x}=r \underline{\omega}, r=|\underline{x}|, \underline{\omega} \in S^{m-1}$.

Taking into account the orthogonality of the Gegenbauer polynomials:

$$
\int_{-1}^{1} t^{\ell} C_{k}^{\lambda}(t)\left(1-t^{2}\right)^{\lambda-1 / 2} d t=0 \text { if } \ell<k
$$

we immediately obtain that in case of $\ell<k$ :

$$
\int_{S^{m-1}}\langle\underline{x}, \underline{t}\rangle^{\ell}(1-i \underline{t}) P_{k}(\underline{t}) d \underline{t}=0
$$

We thus have that

$$
\int_{S^{m-1}} h\left(x_{0}+i\langle\underline{x}, \underline{t}\rangle\right)(1-i \underline{t}) P_{k}(\underline{t}) d \underline{t}=\sum_{\ell=k}^{\infty} \frac{h^{(\ell)}\left(x_{0}\right)}{\ell !} i^{\ell}|\underline{x}|^{\ell} \int_{S^{m-1}}\langle\underline{\omega}, \underline{t}\rangle^{\ell}(1-i \underline{t}) P_{k}(\underline{t}) d \underline{t}
$$


and also

$$
\begin{aligned}
\frac{1}{|\underline{x}|^{k}} C \int_{S^{m-1}} h\left(x_{0}+i\langle\underline{x}, \underline{t}\rangle\right)(1 & -i \underline{t}) P_{k}(\underline{t}) d \underline{t} \\
& =C \sum_{\ell=k}^{\infty} \frac{h^{(\ell)}\left(x_{0}\right) i^{\ell}}{\ell !}|\underline{x}|^{\ell-k} \int_{S^{m-1}}\langle\underline{\omega}, \underline{t}\rangle^{\ell}(1-i \underline{t}) P_{k}(\underline{t}) d \underline{t},
\end{aligned}
$$

from which we immediately see that

$$
\lim _{|\underline{x}| \rightarrow 0} \frac{1}{|\underline{x}|^{k}} f\left(x_{0}, \underline{x}\right)=C \frac{h^{(k)}\left(x_{0}\right) i^{k}}{k !} \int_{S^{m-1}}\langle\underline{\omega}, \underline{t}\rangle^{k}(1-i \underline{t}) P_{k}(\underline{t}) d \underline{t} .
$$

Applying once more the Funk-Hecke theorem gives

$$
\begin{array}{r}
\lim _{|\underline{x}| \rightarrow 0} \frac{1}{|\underline{x}|^{k}} f\left(x_{0}, \underline{x}\right)=C \frac{h^{(k)}\left(x_{0}\right) i^{k}}{k !} \frac{2 \pi^{(m-1) / 2}}{\Gamma\left(\frac{m-1}{2}\right)}\left(P_{k}(\underline{\omega}) \int_{-1}^{1} t^{k}\left(1-t^{2}\right)^{(m-3) / 2} P_{k, m}(t) d t\right. \\
\left.-i \underline{\omega} P_{k}(\underline{\omega}) \int_{-1}^{1} t^{k}\left(1-t^{2}\right)^{(m-3) / 2} P_{k+1, m}(t) d t\right) .
\end{array}
$$

The second integral is zero in view of the orthogonality relation (11). Furthermore, applying the following relation (see [7], p. 188, ex. 4)

$$
\int_{-1}^{1} f(t) P_{k, m}(t)\left(1-t^{2}\right)^{(m-3) / 2} d t=2^{-k} \frac{\Gamma\left(\frac{m-1}{2}\right)}{\Gamma\left(k+\frac{m-1}{2}\right)} \int_{-1}^{1}\left(1-t^{2}\right)^{k+(m-3) / 2} f^{(k)}(t) d t
$$

with $f$ a function which is $k$ times differentiable, we arrive at

$$
\lim _{|\underline{x}| \rightarrow 0} \frac{1}{|\underline{x}|^{k}} f\left(x_{0}, \underline{x}\right)=C \frac{i^{k} 2^{-k+1} \pi^{(m-1) / 2}}{\Gamma\left(k+\frac{m-1}{2}\right)} h^{(k)}\left(x_{0}\right) P_{k}(\underline{\omega}) \int_{-1}^{1}\left(1-t^{2}\right)^{k+(m-3) / 2} d t .
$$

From the orthonormality relation of the Gegenbauer polynomials we derive that

$$
\begin{aligned}
\int_{-1}^{1} C_{0}^{k+(m-2) / 2}(t) C_{0}^{k+(m-2) / 2}(t)\left(1-t^{2}\right)^{k+(m-3) / 2} d t & =\frac{\pi 2^{3-2 k-m} \Gamma(2 k+m-2)}{\left(k+\frac{m-2}{2}\right)\left(\Gamma\left(k+\frac{m-2}{2}\right)\right)^{2}} \\
& =\frac{\sqrt{\pi} \Gamma\left(k+\frac{m}{2}-\frac{1}{2}\right)}{\Gamma\left(k+\frac{m}{2}\right)}
\end{aligned}
$$

where we have used (8). We thus finally obtain

$$
\lim _{|\underline{x}| \rightarrow 0} \frac{1}{|\underline{x}|^{k}} f\left(x_{0}, \underline{x}\right)=C \frac{i^{k} 2^{1-k} \pi^{m / 2}}{\Gamma\left(k+\frac{m}{2}\right)} h^{(k)}\left(x_{0}\right) P_{k}(\underline{\omega}) .
$$

The above calculations lead to the following result. 
Theorem 4.2 Let $h(z)$ be a holomorphic function in $z \in \mathbb{C}, x_{0} \in \mathbb{R}, \underline{x} \in \mathbb{R}^{m}$ and $P_{k}(\underline{t})$ a fixed inner spherical monogenic of degree $k$. Then the function

$$
f\left(x_{0}, \underline{x}\right)=\frac{\Gamma\left(k+\frac{m}{2}\right)(-i)^{k} 2^{k-1}}{\pi^{m / 2}} \int_{S^{m-1}} h\left(x_{0}+i\langle\underline{x}, \underline{t}\rangle\right)(1-i \underline{t}) P_{k}(\underline{t}) d \underline{t}
$$

satisfies

(i)

$$
\left(\partial_{x_{0}}+\partial_{\underline{x}}\right) f\left(x_{0}, \underline{x}\right)=0
$$

(ii)

$$
\lim _{|\underline{x}| \rightarrow 0} \frac{1}{|\underline{x}|^{k}} f\left(x_{0}, \underline{x}\right)=h^{(k)}\left(x_{0}\right) P_{k}(\underline{\omega}), \quad \underline{\omega}=\frac{\underline{x}}{|\underline{x}|} .
$$

In other words, $f\left(x_{0}, \underline{x}\right)$ is the generalized axial CK-extension of $h^{(k)}\left(x_{0}\right)$.

Remark 4.1 Note that we can only link this approach with the generalized CK-extension approach in the axial framework (see Section 2) in case of the function $h\left(x_{0}\right)=e^{x_{0}}$, since only then $h\left(x_{0}\right)=h^{(k)}\left(x_{0}\right)$.

\subsubsection{The biaxial case}

In the biaxial case monogenic plane waves $F(\underline{x}, \underline{y} ; \underline{t}, \underline{s})$ take the form $h(i\langle\underline{x}, \underline{t}\rangle-\langle\underline{y}, \underline{s}\rangle)(\underline{t}+$ $i \underline{s})$ or $h(i\langle\underline{x}, \underline{t}\rangle-\langle\underline{y}, \underline{s}\rangle)(1+i \underline{t s}), \underline{t} \in S^{p-1}, \underline{s} \in S^{q-1}$.

We consider integrals of the form

$$
\int_{S^{p-1}} \int_{S^{q-1}} F(\underline{x}, \underline{y} ; \underline{t}, \underline{s}) P_{k^{\prime}}(\underline{t}) P_{\ell^{\prime}}(\underline{s}) d \underline{t} d \underline{s}
$$

where, as in Section 3, $P_{k^{\prime}}(\underline{x})$ and $P_{\ell^{\prime}}(\underline{y})$ represent solid inner spherical monogenics of degree $k^{\prime}$ in the variable $\underline{x}$ and of degree $\ell^{\prime}$ in the variable $\underline{y}$. Again we assume them to be even Clifford algebra-valued, which implies that they commute.

Integrals of the form (12) lead to biaxial monogenic functions of the form

$$
[A(|\underline{x}|,|\underline{y}|)+\underline{\omega} B(|\underline{x}|,|\underline{y}|)+\underline{\xi} C(|\underline{x}|,|\underline{y}|)+\underline{\omega} \underline{\xi} D(|\underline{x}|,|\underline{y}|)] P_{k^{\prime}}(\underline{\omega}) P_{\ell^{\prime}}(\underline{\xi}), \quad \underline{x}=|\underline{x}| \underline{\omega}, \quad \underline{y}=|\underline{y}| \underline{\xi} .
$$


The evaluation of integrals of the above type is based on applying Funk-Hecke's theorem twice.

Let us first consider the restriction to $\underline{x}=\underline{0}$ of the even Clifford algebra-valued biaxial monogenic:

$$
f^{e}(\underline{x}, \underline{y})=\int_{S^{p-1}} \int_{S^{q-1}} h(i\langle\underline{x}, \underline{t}\rangle-\langle\underline{y}, \underline{s}\rangle)(1+i \underline{t s}) P_{k^{\prime}}(\underline{t}) P_{\ell^{\prime}}(\underline{s}) d \underline{t} d \underline{s} .
$$

Like in the axial case, we start with expanding $h(i\langle\underline{x}, \underline{y}\rangle-\langle\underline{y}, \underline{s}\rangle)$ into a Taylor series:

$$
h(i\langle\underline{x}, \underline{t}\rangle-\langle\underline{y}, \underline{s}\rangle)=\sum_{\ell=0}^{\infty} \frac{(i\langle\underline{x}, \underline{t}\rangle)^{\ell}}{\ell !} h^{(\ell)}(-\langle\underline{y}, \underline{s}\rangle),
$$

which, after application of the Funk-Hecke theorem, leads to

$$
\begin{aligned}
f^{e}(\underline{x}, \underline{y}) & =\sum_{\ell=0}^{\infty} \frac{i^{\ell}}{\ell !}|\underline{x}|^{\ell} \frac{4 \pi^{(p+q) / 2-1}}{\Gamma\left(\frac{p-1}{2}\right) \Gamma\left(\frac{q-1}{2}\right)}\left\{P_{k^{\prime}}(\underline{\omega})\left(\int_{-1}^{1} t^{\ell}\left(1-t^{2}\right)^{(p-3) / 2} P_{k^{\prime}, p}(t) d t\right)\right. \\
& P_{\ell^{\prime}}(\underline{\xi})\left(\int_{-1}^{1} h^{(\ell)}(-|\underline{y}| s)\left(1-s^{2}\right)^{(q-3) / 2} P_{\ell^{\prime}, q}(s) d s\right) \\
& +i \underline{\omega} P_{k^{\prime}}(\underline{\omega})\left(\int_{-1}^{1} t^{\ell}\left(1-t^{2}\right)^{(p-3) / 2} P_{k^{\prime}+1, p}(t) d t\right) \\
& \left.\underline{\xi} P_{\ell^{\prime}}(\underline{\xi})\left(\int_{-1}^{1} h^{(\ell)}(-|\underline{y}| s)\left(1-s^{2}\right)^{(q-3) / 2} P_{\ell^{\prime}+1, q}(s) d s\right)\right\} .
\end{aligned}
$$

From the orthogonality (11) it follows that all terms with $\ell<k^{\prime}$ are zero. Moreover, it is then easily seen that

$$
\begin{array}{r}
\lim _{|\underline{x}| \rightarrow 0} \frac{1}{|\underline{x}|^{k^{\prime}}} f^{e}(\underline{x}, \underline{y})=\frac{i^{k^{\prime}}}{k^{\prime} !} \frac{4 \pi^{(p+q) / 2-1}}{\Gamma\left(\frac{p-1}{2}\right) \Gamma\left(\frac{q-1}{2}\right)} P_{k^{\prime}}(\underline{\omega})\left(\int_{-1}^{1} t^{k^{\prime}}\left(1-t^{2}\right)^{(p-3) / 2} P_{k^{\prime}, p}(t) d t\right) \\
P_{\ell^{\prime}}(\underline{\xi})\left(\int_{-1}^{1} h^{\left(k^{\prime}\right)}(-|\underline{y}| s)\left(1-s^{2}\right)^{(q-3) / 2} P_{\ell^{\prime}, q}(s) d s\right) .
\end{array}
$$

Next, in a similar way as in the axial case, we find that

$$
\int_{-1}^{1} t^{k^{\prime}}\left(1-t^{2}\right)^{(p-3) / 2} P_{k^{\prime}, p}(t) d t=\frac{2^{-k^{\prime}} k^{\prime} ! \sqrt{\pi} \Gamma\left(\frac{p-1}{2}\right)}{\Gamma\left(k^{\prime}+\frac{p}{2}\right)},
$$


hence the boundary value (13) becomes

$$
\begin{aligned}
\lim _{|\underline{\mid x}| \rightarrow 0} & \frac{1}{|\underline{x}|^{k^{\prime}}} f^{e}(\underline{x}, \underline{y}) \\
& =\frac{i^{k^{\prime}} 2^{2-k^{\prime}} \pi^{(p+q-1) / 2}}{\Gamma\left(\frac{q-1}{2}\right) \Gamma\left(k^{\prime}+\frac{p}{2}\right)} P_{k^{\prime}}(\underline{\omega}) P_{\ell^{\prime}}(\underline{\xi})\left(\int_{-1}^{1} h^{\left(k^{\prime}\right)}(-|\underline{y}| s)\left(1-s^{2}\right)^{(q-3) / 2} P_{\ell^{\prime}, q}(s) d s\right) .
\end{aligned}
$$

The Taylor series expansion:

$$
h(i\langle\underline{x}, \underline{t}\rangle-\langle\underline{y}, \underline{s}\rangle)=\sum_{\ell=0}^{\infty} \frac{(-\langle\underline{y}, \underline{s}\rangle)^{\ell}}{\ell !} h^{(\ell)}(i\langle\underline{x}, \underline{t}\rangle),
$$

yields in an analogous way

$$
\begin{aligned}
\lim _{|\underline{y}| \rightarrow 0} & \frac{1}{|\underline{y}|^{\ell^{\prime}}} f^{e}(\underline{x}, \underline{y}) \\
& =\frac{(-1)^{\ell^{\prime}} 2^{2-\ell^{\prime}} \pi^{(p+q-1) / 2}}{\Gamma\left(\frac{p-1}{2}\right) \Gamma\left(\ell^{\prime}+\frac{q}{2}\right)} P_{k^{\prime}}(\underline{\omega}) P_{\ell^{\prime}}(\underline{\xi})\left(\int_{-1}^{1} h^{\left(\ell^{\prime}\right)}(i|\underline{x}| t)\left(1-t^{2}\right)^{(p-3) / 2} P_{k^{\prime}, p}(t) d t\right) .
\end{aligned}
$$

The limit values of the odd Clifford algebra-valued biaxial monogenic:

$$
f^{o}(\underline{x}, \underline{y})=\int_{S^{p-1}} \int_{S^{q-1}} h(i\langle\underline{x}, \underline{t}\rangle-\langle\underline{y}, \underline{s}\rangle)(\underline{t}+i \underline{s}) P_{k^{\prime}}(\underline{t}) P_{\ell^{\prime}}(\underline{s}) d \underline{t} d \underline{s}
$$

take the form

$$
\begin{aligned}
& \lim _{|\underline{x}| \rightarrow 0} \frac{1}{|\underline{x}|^{k^{\prime}}} f^{o}(\underline{x}, \underline{y}) \\
& \quad=\frac{i^{k^{\prime}+1} 2^{2-k^{\prime}} \pi^{(p+q-1) / 2}}{\Gamma\left(\frac{q-1}{2}\right) \Gamma\left(k^{\prime}+\frac{p}{2}\right)} P_{k^{\prime}}(\underline{\omega}) \underline{\xi} P_{\ell^{\prime}}(\underline{\xi})\left(\int_{-1}^{1} h^{\left(k^{\prime}\right)}(-|\underline{y}| s)\left(1-s^{2}\right)^{(q-3) / 2} P_{\ell^{\prime}+1, q}(s) d s\right)
\end{aligned}
$$

and

$$
\begin{aligned}
& \lim _{|\underline{y}| \rightarrow 0} \frac{1}{|\underline{y}|^{\ell^{\prime}}} f^{o}(\underline{x}, \underline{y}) \\
& =\frac{(-1)^{\ell^{\prime}} 2^{2-\ell^{\prime}} \pi^{(p+q-1) / 2}}{\Gamma\left(\frac{p-1}{2}\right) \Gamma\left(\ell^{\prime}+\frac{q}{2}\right)} \underline{\omega} P_{k^{\prime}}(\underline{\omega}) P_{\ell^{\prime}}(\underline{\xi})\left(\int_{-1}^{1} h^{\left(\ell^{\prime}\right)}(i|\underline{x}| t)\left(1-t^{2}\right)^{(p-3) / 2} P_{k^{\prime}+1, p}(t) d t\right) .
\end{aligned}
$$


From Theorem 4.2 it follows that

$$
f\left(x_{0}, \underline{x}\right)=\frac{\Gamma\left(k+\frac{m}{2}\right)(-i)^{k} 2^{k-1}}{\pi^{m / 2}} e^{x_{0}} \int_{S^{m-1}} e^{i\langle\underline{x}, \underline{t}\rangle}(1-i \underline{t}) P_{k}(\underline{t}) d \underline{t}
$$

is the generalized axial CK-extension of $e^{x_{0}}$. From the generalized axial CK-extension approach (see subsection 2.2.1, Remark 2.1) we already know that $f\left(x_{0}, \underline{x}\right)$ takes the form

$$
f\left(x_{0}, \underline{x}\right)=\Gamma\left(\frac{m}{2}+k\right) 2^{m / 2+k-1}\left(J_{m / 2+k-1}(|\underline{x}|)|\underline{x}|^{1-m / 2}+\underline{\omega} J_{m / 2+k}(|\underline{x}|)|\underline{x}|^{1-m / 2}\right) P_{k}(\underline{\omega}) e^{x_{0}} .
$$

We further calculate (18) using the Funk-Hecke theorem

$$
\begin{aligned}
\int_{S^{m-1}} e^{i\langle\underline{x}, \underline{t}\rangle}(1-i \underline{t}) P_{k}(\underline{t}) d \underline{t}=\frac{2 \pi^{(m-1) / 2}}{\Gamma\left(\frac{m-1}{2}\right)}\left(P_{k}(\underline{\omega}) \int_{-1}^{1} e^{i|\underline{x}| t}\left(1-t^{2}\right)^{(m-3) / 2} P_{k, m}(t) d t\right. \\
\left.-i \underline{\omega} P_{k}(\underline{\omega}) \int_{-1}^{1} e^{i|\underline{x}| t}\left(1-t^{2}\right)^{(m-3) / 2} P_{k+1, m}(t) d t\right) .
\end{aligned}
$$

By comparing now the scalar and vector part of (18) and (19) we obtain the integral equations

$$
\begin{aligned}
J_{m / 2+k-1}(|\underline{x}|) & =\frac{(-i)^{k}}{\sqrt{\pi} \Gamma\left(\frac{m-1}{2}\right)}\left(\frac{|\underline{x}|}{2}\right)^{m / 2-1} \int_{-1}^{1} e^{i|\underline{x}| t}\left(1-t^{2}\right)^{(m-3) / 2} P_{k, m}(t) d t \\
J_{m / 2+k}(|\underline{x}|) & =\frac{(-i)^{k+1}}{\sqrt{\pi} \Gamma\left(\frac{m-1}{2}\right)}\left(\frac{|\underline{x}|}{2}\right)^{m / 2-1} \int_{-1}^{1} e^{i|\underline{x}| t}\left(1-t^{2}\right)^{(m-3) / 2} P_{k+1, m}(t) d t
\end{aligned}
$$

which are an application of the following integral expression which is derived in [7], p. 203-204:

$$
J_{n+m / 2-1}(z)=\frac{(-i)^{n}}{\sqrt{\pi} \Gamma\left(\frac{m-1}{2}\right)}\left(\frac{z}{2}\right)^{m / 2-1} \int_{-1}^{1} e^{i z t} P_{n, m}(t)\left(1-t^{2}\right)^{(m-3) / 2} d t .
$$


We will now illustrate the monogenic plane wave approach in the biaxial framework for $h(z)=e^{z}$ in order to obtain integral formulas for the Clifford-Bessel function of biaxial type (see subsection 3.2, Remark 3.1):

$$
\begin{aligned}
f^{B e s}(\underline{x}, \underline{y})=2^{q / 2+\ell^{\prime}-1} \Gamma\left(\frac{q}{2}+\ell^{\prime}\right)\left(\widetilde{J}_{q / 2+\ell^{\prime}-1}(i|\underline{y}|)-\widetilde{J}_{q / 2+\ell^{\prime}}(i|\underline{y}|) \underline{y}\right) \\
\\
\quad \times\left(\widetilde{J}_{k^{\prime}+p / 2-1}(|\underline{x}|)+\underline{x} \widetilde{J}_{k^{\prime}+p / 2}(|\underline{x}|)\right) P_{k^{\prime}}(\underline{x}) P_{\ell^{\prime}}(\underline{y}) .
\end{aligned}
$$

Applying Funk-Hecke twice, we obtain for the odd Clifford algebra-valued biaxial monogenic

$$
\begin{aligned}
f^{o}(\underline{x}, \underline{y}) & =\int_{S^{p-1}} \int_{S^{q-1}} e^{i\langle\underline{x}, \underline{t}\rangle-\langle\underline{y}, \underline{s}\rangle}(\underline{t}+i \underline{s}) P_{k^{\prime}}(\underline{t}) P_{\ell^{\prime}}(\underline{s}) d \underline{t} d \underline{s} \\
& =\frac{4 \pi^{(p+q) / 2-1}}{\Gamma\left(\frac{p-1}{2}\right) \Gamma\left(\frac{q-1}{2}\right)} \underline{\omega} P_{k^{\prime}}(\underline{\omega})\left(\int_{-1}^{1} e^{i|\underline{x}| t}\left(1-t^{2}\right)^{(p-3) / 2} P_{k^{\prime}+1, p}(t) d t\right) \\
& \times P_{\ell^{\prime}}(\underline{\xi})\left(\int_{-1}^{1} e^{-|\underline{y}| s}\left(1-s^{2}\right)^{(q-3) / 2} P_{\ell^{\prime}, q}(s) d s\right) \\
& +i \frac{4 \pi}{\Gamma\left(\frac{p-1}{2}\right) \Gamma\left(\frac{q-1}{2}\right)} P_{k^{\prime}}(\underline{\omega})\left(\int_{-1}^{1} e^{i|\underline{x}| t}\left(1-t^{2}\right)^{(p-3) / 2} P_{k^{\prime}, p}(t) d t\right) \\
& \times \underline{\xi} P_{\ell^{\prime}}(\underline{\xi})\left(\int_{-1}^{1} e^{-|\underline{y}| s}\left(1-s^{2}\right)^{(q-3) / 2} P_{\ell^{\prime}+1, q}(s) d s\right) .
\end{aligned}
$$

Next, in view of (20) we become

$$
\begin{array}{r}
f^{o}(\underline{x}, \underline{y})=i^{k^{\prime}+1}(-1)^{\ell^{\prime}}(2 \pi)^{(p+q) / 2}\left(\widetilde{J}_{k^{\prime}+p / 2}(|\underline{x}|) \widetilde{J}_{\ell^{\prime}+q / 2-1}(i|\underline{y}|) \underline{x}-\widetilde{J}_{k^{\prime}+p / 2-1}(|\underline{x}|) \widetilde{J}_{\ell^{\prime}+q / 2}(i|\underline{y}|) \underline{y}\right) \\
\times P_{k^{\prime}}(\underline{x}) P_{\ell^{\prime}}(\underline{y}) .
\end{array}
$$

In a similar way, we find for the even Clifford algebra-valued biaxial monogenic:

$$
\begin{aligned}
f^{e}(\underline{x}, \underline{y}) & =\int_{S^{p-1}} \int_{S^{q-1}} e^{i\langle\underline{x}, \underline{t}\rangle-\langle\underline{y}, \underline{s}\rangle}(1+i \underline{t s}) P_{k^{\prime}}(\underline{t}) P_{\ell^{\prime}}(\underline{s}) d \underline{t} d \underline{s} \\
& =i^{k^{\prime}}(-1)^{\ell^{\prime}}(2 \pi)^{(p+q) / 2}\left(\widetilde{J}_{k^{\prime}+p / 2-1}(|\underline{x}|) \widetilde{J}_{\ell^{\prime}+q / 2-1}(i|\underline{y}|)+\underline{x} \underline{y} \widetilde{J}_{k^{\prime}+p / 2}(|\underline{x}|) \widetilde{J}_{\ell^{\prime}+q / 2}(i|\underline{y}|)\right) \\
& \times P_{k^{\prime}}(\underline{x}) P_{\ell^{\prime}}(\underline{y}) .
\end{aligned}
$$


From the above calculations and subsection 3.2, we obtain the following result.

Theorem 4.3 The odd and even Clifford algebra-valued part of the Clifford-Bessel function of biaxial type

$$
\begin{aligned}
f^{B e s}(\underline{x}, \underline{y})=2^{q / 2+\ell^{\prime}-1} \Gamma\left(\frac{q}{2}+\ell^{\prime}\right)\left(\widetilde{J}_{q / 2+\ell^{\prime}-1}(i|\underline{y}|)-\widetilde{J}_{q / 2+\ell^{\prime}}(i|\underline{y}|) \underline{y}\right) & \\
& \times\left(\widetilde{J}_{k^{\prime}+p / 2-1}(|\underline{x}|)+\underline{x} \widetilde{J}_{k^{\prime}+p / 2}(|\underline{x}|)\right) P_{k^{\prime}}(\underline{x}) P_{\ell^{\prime}}(\underline{y}) .
\end{aligned}
$$

can be written as plane wave integrals:

$$
\begin{aligned}
& {\left[f^{B e s}(\underline{x}, \underline{y})\right]_{o}=\frac{(-1)^{\ell^{\prime}}(-i)^{k^{\prime}+1} \Gamma\left(\frac{q}{2}+\ell^{\prime}\right)}{2^{1+p / 2-\ell^{\prime}} \pi^{(p+q) / 2}} \int_{S^{p-1}} \int_{S^{q-1}} e^{i\langle\underline{x}, \underline{t}\rangle-\langle\underline{y}, \underline{s}\rangle}(\underline{t}+i \underline{s}) P_{k^{\prime}}(\underline{t}) P_{\ell^{\prime}}(\underline{s}) d \underline{t} d \underline{s}} \\
& {\left[f^{B e s}(\underline{x}, \underline{y})\right]_{e}=\frac{(-1)^{\ell^{\prime}}(-i)^{k^{\prime}} \Gamma\left(\frac{q}{2}+\ell^{\prime}\right)}{2^{1+p / 2-\ell^{\prime}} \pi^{(p+q) / 2}} \int_{S^{p-1}} \int_{S^{q-1}} e^{i\langle\underline{x}, \underline{t}\rangle-\langle\underline{y}, \underline{s}\rangle}(1+i \underline{t s}) P_{k^{\prime}}(\underline{t}) P_{\ell^{\prime}}(\underline{s}) d \underline{t} d \underline{s} .}
\end{aligned}
$$

One thus also has that

$f^{B e s}(\underline{x}, \underline{y})=\frac{(-1)^{\ell^{\prime}}(-i)^{k^{\prime}} \Gamma\left(\frac{q}{2}+\ell^{\prime}\right)}{2^{1+p / 2-\ell^{\prime}} \pi^{(p+q) / 2}} \int_{S^{p-1}} \int_{S^{q-1}} e^{i\langle\underline{x}, \underline{t}\rangle-\langle\underline{y}, \underline{s}\rangle}(1-i \underline{t}+\underline{s}+i \underline{t s}) P_{k^{\prime}}(\underline{t}) P_{\ell^{\prime}}(\underline{s}) d \underline{t} d \underline{s}$.

The boundary values (14), (15), (16) and (17) can easily be calculated using (see (20)):

$$
\int_{-1}^{1} e^{i z t}\left(1-t^{2}\right)^{(m-3) / 2} P_{n, m}(t) d t=\sqrt{\pi} \Gamma\left(\frac{m-1}{2}\right) i^{n}\left(\frac{2}{z}\right)^{m / 2-1} J_{n+m / 2-1}(z) .
$$

We find

$$
\begin{aligned}
& \lim _{|\underline{x}| \rightarrow 0} \frac{1}{|\underline{x}|^{k^{\prime}}} f^{e}(\underline{x}, \underline{y})=\frac{(-1)^{\ell^{\prime}} i^{k^{\prime}} 2^{1-k^{\prime}+q / 2} \pi^{(p+q) / 2}}{\Gamma\left(k^{\prime}+\frac{p}{2}\right)} \widetilde{J}_{\ell^{\prime}+q / 2-1}(i|\underline{y}|) P_{k^{\prime}}(\underline{\omega}) P_{\ell^{\prime}}(\underline{y}) \\
& \lim _{|\underline{y}| \rightarrow 0} \frac{1}{|\underline{y}|^{\ell^{\prime}}} f^{e}(\underline{x}, \underline{y})=\frac{(-1)^{\ell^{\prime}} i^{k^{\prime}} 2^{1-\ell^{\prime}+p / 2} \pi^{(p+q) / 2}}{\Gamma\left(\ell^{\prime}+\frac{q}{2}\right)} \widetilde{J}_{k^{\prime}+p / 2-1}(|\underline{x}|) P_{k^{\prime}}(\underline{x}) P_{\ell^{\prime}}(\underline{\xi}) \\
& \lim _{|\underline{x}| \rightarrow 0} \frac{1}{|\underline{x}|^{k^{\prime}}} f^{o}(\underline{x}, \underline{y})=\frac{(-1)^{\ell^{\prime}+1} i^{k^{\prime}+1} 2^{1-k^{\prime}+q / 2} \pi^{(p+q) / 2}}{\Gamma\left(k^{\prime}+\frac{p}{2}\right)} \widetilde{J}_{\ell^{\prime}+q / 2}(i|\underline{y}|) P_{k^{\prime}}(\underline{\omega}) \underline{y} P_{\ell^{\prime}}(\underline{y}) \\
& \lim _{|\underline{y}| \rightarrow 0} \frac{1}{|\underline{y}|^{\ell^{\prime}}} f^{o}(\underline{x}, \underline{y})=\frac{(-1)^{\ell^{\prime}} i^{k^{\prime}+1} 2^{1-\ell^{\prime}+p / 2} \pi^{(p+q) / 2}}{\Gamma\left(\ell^{\prime}+\frac{q}{2}\right)} \widetilde{J}_{k^{\prime}+p / 2}(|\underline{x}|) \underline{x} P_{k^{\prime}}(\underline{x}) P_{\ell^{\prime}}(\underline{\xi}) .
\end{aligned}
$$




\section{References}

[1] F. Brackx, R. Delanghe, F. Sommen, Clifford analysis, vol. 76 of Research Notes in Mathematics, Pitman (Advanced Publishing Program), Boston, MA, 1982.

[2] F. Colombo, I. Sabadini, F. Sommen, The inverse Fueter mapping theorem in integral form using spherical monogenics, accepted for publication in Israel Journal of Mathematics.

[3] R. Delanghe, F. Sommen, V. Souček, Clifford algebra and spinor-valued functions, vol. 53 of Mathematics and its Applications, Kluwer Academic Publishers Group, Dordrecht, 1992.

[4] N. De Schepper, F. Sommen, Cauchy-Kowelevski extensions and monogenic plane waves in Clifford analysis, accepted for publication in Adv. Appl. Clifford Alg. 22(3) (2012) 625-647.

[5] J. Gilbert, M. Murray, Clifford Algebras and Dirac Operators in Harmonic Analysis, Cambridge University Press, Cambridge, 1991.

[6] K. Gürlebeck, K. Habetha, W. Sprössig, Holomorphic Functions in the Plane and ndimensional Space, Birkhäuser, Basel, 2008.

[7] H. Hochstadt, The Functions of Mathematical Physics, in: "Pure and Applied Mathematics", vol. 23, Wiley-Interscience, New York, 1971.

[8] G. Jank, F. Sommen, Clifford analysis, biaxial symmetry and pseudoanalytic functions, Complex Var. Elliptic Equ. 13(3-4) (1990) 195-212.

[9] R. Lávička, A generalization of Fueter's monogenic functions to fine domains, Rend. Circ. Mat. di Palermo (2) Suppl. 79 (2006) 129-138.

[10] W. Magnus, F. Oberhettinger, R. P. Soni, Formulas and Theorems for the Special Functions of Mathematical Physics, Springer-Verlag, Berlin, 1966.

[11] D. Peña Peña, T. Qian, F. Sommen, An alternative proof of Fueter's theorem, Complex Var. Elliptic Equ. 51(8-11) (2006) 913-922. 
[12] D. Peña Peña, F. Sommen, Monogenic Gaussian distribution in closed form and the Gaussian fundamental solution, Complex Var. Elliptic Equ. 54, no. 5 (2009) 429-440.

[13] T. Qian, F. Sommen, Deriving harmonic functions in higher dimensional spaces, Z. Anal. Anwendungen 22(2) (2003) 275-288.

[14] F. Sommen, A product and an exponential function in hypercomplex function theory, Applicable Analysis 12 (1981) 13-26.

[15] F. Sommen, Special Functions in Clifford analysis and Axial Symmetry, J. Math. Anal. Appl. 130, no. 1 (1988) 110-133.

[16] F. Sommen, Clifford analysis and integral geometry, in: "Clifford Algebras and their Applications in Mathematical Physics", A. Micali et al., Kluwer Academic Publishers (1992) 293-311. 